\title{
Stochastic Homogenisation of Free-Discontinuity Problems
}

\author{
Filippo Cagnetti, Gianni Dal Maso, Lucia Scardia@ \& \\ CATERINA IDA ZEPPIERI
}

Communicated by A. BRAIDES

\begin{abstract}
In this paper we study the stochastic homogenisation of free-discontinuity functionals. Assuming stationarity for the random volume and surface integrands, we prove the existence of a homogenised random free-discontinuity functional, which is deterministic in the ergodic case. Moreover, by establishing a connection between the deterministic convergence of the functionals at any fixed realisation and the pointwise Subadditive Ergodic Theorem by Akcoglou and Krengel, we characterise the limit volume and surface integrands in terms of asymptotic cell formulas.
\end{abstract}

\section{Introduction}

In this article we prove a stochastic homogenisation result for sequences of free-discontinuity functionals of the form

$$
E_{\varepsilon}(\omega)(u)=\int_{A} f\left(\omega, \frac{x}{\varepsilon}, \nabla u\right) \mathrm{d} x+\int_{S_{u} \cap A} g\left(\omega, \frac{x}{\varepsilon}, u^{+}-u^{-}, v_{u}\right) \mathrm{d} \mathcal{H}^{n-1},
$$

where $f$ and $g$ are random integrands, $\omega$ is the random parameter, and $\varepsilon>0$ is a small scale parameter. The functionals $E_{\varepsilon}$ are defined in the space $S B V\left(A, \mathbb{R}^{m}\right)$ of special $\mathbb{R}^{m}$-valued functions of bounded variation on the open set $A \subset \mathbb{R}^{n}$. This space was introduced by De Giorgi and Ambrosio in [22] to deal with deterministic problems - for example in fracture mechanics, image segmentation, or in the study of liquid crystals - where the variable $u$ can have discontinuities on a hypersurface which is not known a priori, hence the name free-discontinuity functionals [21]. In (1.1), $S_{u}$ denotes the discontinuity set of $u, u^{+}$and $u^{-}$are the "traces" of $u$ on both sides of $S_{u}, v_{u}$ denotes the (generalised) normal to $S_{u}$, and $\nabla u$ denotes the approximate differential of $u$. 
Our main result is that, in the macroscopic limit $\varepsilon \rightarrow 0$, the functionals $E_{\varepsilon}$ homogenise to a stochastic free-discontinuity functional of the same form, under the assumption that $f$ and $g$ are stationary with respect to $\omega$, and that each of the realisations $f(\omega, \cdot, \cdot)$ and $g(\omega, \cdot, \cdot, \cdot)$ satisfies the hypotheses considered in the deterministic case studied in [16] (see Section 3 for details). Moreover, we show that under the additional assumption of ergodicity of $f$ and $g$ the homogenised limit of $E_{\varepsilon}$ is deterministic. Therefore, our qualitative homogenisation result extends to the $S B V$-setting the classical qualitative results by PAPANICOLAOU and VARADHAN [31,32], KozLov [28], and DAL MAso and Modica [17, 18], which were formulated in the more regular Sobolev setting.

\subsection{A Brief Literature Review}

The study of variational limits of random free-discontinuity functionals is very much at its infancy. To date, the only available results are limited to the special case of discrete energies of spin systems $[2,14]$, where the authors consider purely surface integrals, and $u$ is defined on a discrete lattice and takes values in $\{ \pm 1\}$.

In the case of volume functionals in Sobolev spaces, classical qualitative results are provided by the work by PAPANICOLAOU and VARADHAN [31,32] and KozLOV [28] in the linear case, and by DAL Maso and Modica $[17,18]$ in the nonlinear setting. The need to develop efficient methods to determine the homogenised coefficients and to estimate the error in the homogenisation approximation, has recently motivated an intense effort to build a quantitative theory of stochastic homogenisation in the regular Sobolev case.

The first results in this direction are due to Gloria and Otto in the discrete setting [26,27]. In the continuous setting, quantitative estimates for the convergence results are given by ARMSTRONG and SMART [8], who also study the regularity of the minimisers, and by Armstrong, KuUsi, and Mourrat [5,6]. We also mention [7], where Armstrong and Mourrat give Lipschitz regularity for the solutions of elliptic equations with random coefficients, by directly studying certain functionals that are minimised by the solutions.

The mathematical theory of deterministic homogenisation of free-discontinuity problems is well established. When $f$ and $g$ are periodic in the spatial variable, the limit behaviour of $E_{\varepsilon}$ can be determined by classical homogenisation theory. In this case, under mild assumptions on $f$ and $g$, the deterministic functionals $E_{\varepsilon}$ behave macroscopically like a homogeneous free-discontinuity functional. If, in addition, the integrands $f$ and $g$ satisfy some standard growth and coercivity conditions, the limit behaviour of $E_{\varepsilon}$ is given by the simple superposition of the limit behaviours of its volume and surface parts (see [13]). This is, however, not always the case if $f$ and $g$ satisfy "degenerate" coercivity conditions. Indeed, while in $[10,15,25]$ the two terms in $E_{\varepsilon}$ do not interact, in [9,11,20,33-35] they do interact and produce rather complex limit effects. The study of the deterministic homogenisation of free-discontinuity functionals without any periodicity condition, and under general assumptions ensuring that the volume and surface terms do "not mix" in the limit, has been recently carried out in [16]. 


\subsection{Stationary Random Integrands}

Before giving the precise statement of our results, we need to recall some definitions. The random environment is modelled by a probability space $(\Omega, \mathcal{T}, P)$ endowed with a group $\tau=\left(\tau_{z}\right)_{z \in \mathbb{Z}^{n}}$ (resp. $\left.\tau=\left(\tau_{z}\right)_{z \in \mathbb{R}^{n}}\right)$ of $\mathcal{T}$-measurable $P$ preserving transformations on $\Omega$. That is, the action of $\tau$ on $\Omega$ satisfies

$$
P(\tau(E))=P(E) \text { for every } E \in \mathcal{T} \text {. }
$$

We say that $f: \Omega \times \mathbb{R}^{n} \times \mathbb{R}^{m \times n} \rightarrow[0,+\infty)$ and $g: \Omega \times \mathbb{R}^{n} \times\left(\mathbb{R}^{m} \backslash\{0\}\right) \times$ $\mathbb{S}^{n-1} \rightarrow[0,+\infty)$ are stationary random volume and surface integrands if they satisfy the assumptions introduced in the deterministic work [16] (see Section 3 for the complete list of assumptions) for every realisation, and the following stationarity condition with respect to $\tau$ : for every $z \in \mathbb{Z}^{n}$ (resp. $z \in \mathbb{R}^{n}$ ) we have

$$
\begin{aligned}
f(\omega, x+z, \xi) & =f\left(\tau_{z}(\omega), x, \xi\right) & & \text { for every }(x, \xi) \in \mathbb{R}^{n} \times \mathbb{R}^{m \times n} \\
g(\omega, x+z, \zeta, v) & =g\left(\tau_{z}(\omega), x, \zeta, v\right) & & \text { for every }(x, \zeta, v) \in \mathbb{R}^{n} \times \mathbb{R}_{0}^{m} \times \mathbb{S}^{n-1}
\end{aligned}
$$

When, in addition, $\tau$ is ergodic, namely when any $\tau$-invariant set $E \in \mathcal{T}$ has probability zero or one, we say that $f$ and $g$ are ergodic.

\subsection{The Main Result: Method of Proof and Comparison with Previous Works}

Under the assumption that $f$ and $g$ are stationary random integrands, we prove the convergence of $E_{\varepsilon}$ to a random homogenised functional $E_{\text {hom }}$ (Theorem 3.13), and we provide representation formulas for the limit volume and surface integrands (Theorem 3.12). The combination of these two results shows, in particular, that the limit functional $E_{\mathrm{hom}}$ is a free-discontinuity functional of the same form as $E_{\varepsilon}$. If, in addition, $f$ and $g$ are ergodic, we show that $E_{\text {hom }}$ is deterministic.

Our method of proof consists of two main steps: a purely deterministic step and a stochastic one, in the spirit of the strategy introduced in [18] for integral functionals of volume type defined on Sobolev spaces.

In the deterministic step we fix $\omega \in \Omega$ and we study the asymptotic behaviour of $E_{\varepsilon}(\omega)$. Our recent result [16, Theorem 3.11] ensures that $E_{\varepsilon}(\omega)$ converges (in the sense of $\Gamma$-convergence) to a free-discontinuity functional of the form

$$
E_{\mathrm{hom}}(\omega)(u)=\int_{A} f_{\text {hom }}(\omega, \nabla u) \mathrm{d} x+\int_{S_{u} \cap A} g_{\text {hom }}\left(\omega,[u], v_{u}\right) \mathrm{d} \mathcal{H}^{n-1},
$$

with

$$
\begin{aligned}
f_{\mathrm{hom}}(\omega, \xi) & :=\lim _{r \rightarrow 0^{+}} \frac{1}{r^{n}} \inf \int_{Q_{r}(r x)} f(\omega, y, \nabla u(y)) \mathrm{d} y, \\
g_{\text {hom }}(\omega, \zeta, v) & :=\lim _{r \rightarrow 0^{+}} \frac{1}{r^{n-1}} \inf \int_{S_{u} \cap Q_{r}^{v}(r x)} g\left(\omega, y,[u](y), v_{u}(y)\right) \mathrm{d} \mathcal{H}^{n-1}(y),
\end{aligned}
$$


provided the limits in (1.2)-(1.3) exist and are independent of $x$. In (1.2) the infimum is taken among Sobolev functions attaining the linear boundary datum $\xi x$ near $\partial Q_{r}(r x)$ (see (1.4) below), where $Q_{r}(r x)=r Q(x)$ is the blow-up by $r$ of the unit cube centred at $x$. In (1.3) the infimum is taken among all Caccioppoli partitions (namely $u \in S B V_{\mathrm{pc}}\left(Q_{r}^{v}(r x), \mathbb{R}^{m}\right)$, see (f) in Section 2) attaining a piecewise constant boundary datum near $\partial Q_{r}^{v}(r x)$ (see (1.5)), and $Q_{r}^{v}(r x)$ is obtained by rotating $Q_{r}(r x)$ in such a way that one face is perpendicular to $v$.

In the stochastic step we prove that the limits (1.2) and (1.3) exist almost surely and are independent of $x$. To this end, it is crucial to show that we can apply the Subadditive Ergodic Theorem by AKcoglou and Krengel [1]. Since our convergence result [16] ensures that there is no interaction between the volume and surface terms in the limit, we can treat them separately.

More precisely, for the volume term, proceeding as in [18] (see also [30]), one can show that the map

$(\omega, Q) \mapsto \inf \left\{\int_{Q} f(\omega, y, \nabla u(y)) \mathrm{d} y: u \in W^{1, p}\left(Q, \mathbb{R}^{m}\right), u(y)=\xi y\right.$ near $\left.\partial Q\right\}$

defines a subadditive stochastic process for every fixed $\xi \in \mathbb{R}^{m \times n}$ (see Definition 3.10). Then the almost sure existence of the limit of (1.2) and its independence of $x$ directly follow from the $n$-dimensional pointwise Subadditive Ergodic Theorem, which also ensures that the limit is deterministic if $f$ is ergodic.

For the surface term, however, applying this general programme presents several difficulties. One of the obstacles is due to a nontrivial "mismatch" of dimensions: on the one hand the minimisation problem

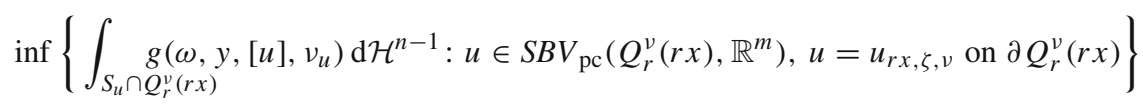

appearing in (1.3) is defined on the $n$-dimensional set $Q_{r}^{v}(r x)$; on the other hand the integration is performed on the $(n-1)$-dimensional set $S_{u} \cap Q_{r}^{v}(r x)$ and the integral rescales in $r$ like a surface measure. In other words, the surface term is an $(n-1)$-dimensional measure which is naturally defined on $n$-dimensional sets. Understanding how to match these different dimensions is a key preliminary step to define a suitable subadditive stochastic process for the application of the Subadditive Ergodic Theorem in dimension $n-1$.

To this end we first set $x=0$. We want to consider the infimum in (1.5) as a function of $(\omega, I)$, where $I$ belongs to the class $\mathcal{I}_{n-1}$ of $(n-1)$-dimensional intervals (see (3.9)). To do so, we define a systematic way to "complete" the missing dimension and to rotate the resulting $n$-dimensional interval. For this we proceed as in [2], where the authors had to face a similar problem in the study of pure surface energies of spin systems. 
Once this preliminary problem is overcome, we prove in Proposition 5.3 that the infimum in (1.5) with $x=0$ and $v$ with rational coordinates is related to an $(n-1)$-dimensional subadditive stochastic process $\mu_{\zeta, v}$ on $\Omega \times \mathcal{I}_{n-1}$ with respect to a suitable group $\left(\tau_{z^{\prime}}^{\nu}\right)_{z^{\prime} \in \mathbb{Z}^{n-1}}\left(\operatorname{resp} .\left(\tau_{z^{\prime}}^{\nu}\right)_{z^{\prime} \in \mathbb{R}^{n-1}}\right)$ of $P$-preserving transformations (see Proposition 5.3). A key difficulty in the proof is to establish the measurability in $\omega$ of the infimum (1.5). Note that this is clearly not an issue in the case of volume integrals considered in [17,18]: The infimum in (1.4) is computed on a separable space, so it can be done over a countable set of functions, and hence the measurability of the process follows directly from the measurability of $f$. This is not an issue for the surface energies considered in [2] either; since the problem is studied in a discrete lattice, the minimisation is reduced to a countable collection of functions. The infimum in (1.5), instead, cannot be reduced to a countable set, hence the proof of measurability is not straightforward (see Proposition A.1 in the Appendix).

The next step is to apply the $(n-1)$-dimensional Subadditive Ergodic Theorem to the subadditive stochastic process $\mu_{\zeta, v}$, for fixed $\zeta$ and $\nu$. This ensures that the limit

$$
g_{\zeta, v}(\omega):=\lim _{t \rightarrow+\infty} \frac{\mu_{\zeta, v}(\omega)(t I)}{t^{n-1} \mathcal{L}^{n-1}(I)}
$$

exists for $P$-almost everywhere $\omega \in \Omega$ and does not depend on $I$. The fact that the limit in (1.6) exists in a set of full measure, common to every $\zeta$ and $v$, requires some attention (see Proposition 5.1), and follows from the continuity properties in $\zeta$ and $v$ of some auxiliary functions (see (5.10) and (5.11) in Lemma 5.5).

As a final step, we need to show that the limit in (1.3) is independent of $x$, namely that the choice $x=0$ is not restrictive. We remark that the analogous result for (1.2) follows directly by $\Gamma$-convergence and by the Subadditive Ergodic Theorem (see also [18]). The surface case, however, is more subtle, since the minimisation problem in (1.5) depends on $x$ also through the boundary datum $u_{r x, \zeta, v}$. To prove the $x$-independence of $g_{\text {hom }}$ we proceed in three steps. First, we exploit the stationarity of $g$ to show that (1.6) is $\tau$-invariant. Then, we prove the result when $x$ is integer, by combinining the Subadditive Ergodic Theorem and the Birkhoff Ergodic Theorem, in the spirit of [2, Proof of Theorem 5.5] (see also [14, Proposition 2.10]). Finally, we conclude the proof with a careful approximation argument.

\subsection{Outline of the Paper}

The paper is organised as follows: in Section 2 we introduce some notation used throughout the paper. In the first part of Section 3 we state the assumptions on $f$ and $g$ and we introduce the stochastic setting of the problem; the second part is devoted to the statement of the main results of the paper. The behaviour of the volume term is studied in the short Section 4, while Sections 5 and 6, as well as the Appendix, deal with the surface term. 


\section{Notation}

We introduce now some notation that will be used throughout the paper. For the convenience of the reader we follow the ordering used in [16]:

(a) $m$ and $n$ are fixed positive integers, with $n \geq 2, \mathbb{R}$ is the set of real numbers, and $\mathbb{R}_{0}^{m}:=\mathbb{R}^{m} \backslash\{0\}$, while $\mathbb{Q}$ is the set of rational numbers and $\mathbb{Q}_{0}^{m}:=\mathbb{Q}^{m} \backslash\{0\}$. The canonical basis of $\mathbb{R}^{n}$ is denoted by $e_{1}, \ldots, e_{n}$. For $a, b \in \mathbb{R}^{n}, a \cdot b$ denotes the Euclidean scalar product between $a$ and $b$, and $|\cdot|$ denotes the absolute value in $\mathbb{R}$ or the Euclidean norm in $\mathbb{R}^{n}, \mathbb{R}^{m}$, or $\mathbb{R}^{m \times n}$, depending on the context.

(b) $\mathbb{S}^{n-1}:=\left\{x=\left(x_{1}, \ldots, x_{n}\right) \in \mathbb{R}^{n}: x_{1}^{2}+\cdots+x_{n}^{2}=1\right\}, \mathbb{S}_{ \pm}^{n-1}:=\{x \in$ $\left.\mathbb{S}^{n-1}: \pm x_{n}>0\right\}$, and $\widehat{\mathbb{S}}_{ \pm}^{n-1}:=\left\{x \in \mathbb{S}^{n-1}: \pm x_{i(x)}>0\right\}$, where $i(x)$ is the largest $i \in\{1, \ldots, n\}$ such that $x_{i} \neq 0$. Note that $\mathbb{S}_{ \pm}^{n-1} \subset \widehat{\mathbb{S}}_{ \pm}^{n-1}$, and that $\mathbb{S}^{n-1}=\widehat{\mathbb{S}}_{+}^{n-1} \cup \widehat{\mathbb{S}}_{-}^{n-1}$.

(c) $\mathcal{L}^{n}$ denotes the Lebesgue measure on $\mathbb{R}^{n}$ and $\mathcal{H}^{n-1}$ the $(n-1)$-dimensional Hausdorff measure on $\mathbb{R}^{n}$.

(d) $\mathscr{A}$ denotes the collection of all bounded open subsets of $\mathbb{R}^{n}$; if $A, B \in \mathscr{A}$, by $A \subset \subset B$ we mean that $A$ is relatively compact in $B$.

(e) For $u \in G S B V\left(A, \mathbb{R}^{m}\right)$ (see [4, Section 4.5]), with $A \in \mathscr{A}$, the jump of $u$ across $S_{u}$ is defined by $[u]:=u^{+}-u^{-}$.

(f) For $A \in \mathscr{A}$ we define

$S B V_{\mathrm{pc}}\left(A, \mathbb{R}^{m}\right):=\left\{u \in S B V\left(A, \mathbb{R}^{m}\right): \nabla u=0 \mathcal{L}^{n}\right.$-almost everywhere,

$$
\left.\mathcal{H}^{n-1}\left(S_{u}\right)<+\infty\right\}
$$

(g) For $A \in \mathscr{A}$ and $p>1$ we define

$$
\begin{array}{r}
S B V^{p}\left(A, \mathbb{R}^{m}\right):=\left\{u \in S B V\left(A, \mathbb{R}^{m}\right): \nabla u \in L^{p}\left(A, \mathbb{R}^{m \times n}\right),\right. \\
\left.\mathcal{H}^{n-1}\left(S_{u}\right)<+\infty\right\} .
\end{array}
$$

(h) For $A \in \mathscr{A}$ and $p>1$ we define

$$
\begin{array}{r}
G S B V^{p}\left(A, \mathbb{R}^{m}\right):=\left\{u \in G S B V\left(A, \mathbb{R}^{m}\right): \nabla u \in L^{p}\left(A, \mathbb{R}^{m \times n}\right),\right. \\
\left.\mathcal{H}^{n-1}\left(S_{u}\right)<+\infty\right\} ;
\end{array}
$$

it is known that $G S B V^{p}\left(A, \mathbb{R}^{m}\right)$ is a vector space and that for every $u \in$ $G S B V^{p}\left(A, \mathbb{R}^{m}\right)$ and for every $\psi \in C_{c}^{1}\left(\mathbb{R}^{m}, \mathbb{R}^{m}\right)$ we have $\psi(u) \in S B V^{p}$ $\left(A, \mathbb{R}^{m}\right) \cap L^{\infty}\left(A, \mathbb{R}^{m}\right)$ (see, for example, [19, page 172]).

(i) For every $\mathcal{L}^{n}$-measurable set $A \subset \mathbb{R}^{n}$ let $L^{0}\left(A, \mathbb{R}^{m}\right)$ be the space of all $\left(\mathcal{L}^{n}\right.$ equivalence classes of) $\mathcal{L}^{n}$-measurable functions $u: A \rightarrow \mathbb{R}^{m}$, endowed with the topology of convergence in measure on bounded subsets of $A$; we observe that this topology is metrisable and separable.

(j) For $x \in \mathbb{R}^{n}$ and $\rho>0$ we define

$$
\begin{aligned}
& B_{\rho}(x):=\left\{y \in \mathbb{R}^{n}:|y-x|<\rho\right\}, \\
& Q_{\rho}(x):=\left\{y \in \mathbb{R}^{n}:\left|(y-x) \cdot e_{i}\right|<\rho / 2 \text { for } i=1, \ldots, n\right\} .
\end{aligned}
$$

We omit the subscript $\rho$ when $\rho=1$. 
(k) For every $v \in \mathbb{S}^{n-1}$ let $R_{\nu}$ be an orthogonal $n \times n$ matrix such that $R_{\nu} e_{n}=v$; we assume that the restrictions of the function $v \mapsto R_{v}$ to the sets $\widehat{\mathbb{S}}_{+}^{n-1}$ defined in (b) are continuous and that $R_{-\nu} Q(0)=R_{\nu} Q(0)$ for every $v \in \mathbb{S}^{n-1}$; moreover, we assume that $R_{v} \in O(n) \cap \mathbb{Q}^{n \times n}$ for every $v \in \mathbb{Q}^{n} \cap \mathbb{S}^{n-1}$. A map $v \mapsto R_{v}$ satisfying these properties is provided in [16, Example A.1 and Remark A.2].

(1) For $x \in \mathbb{R}^{n}, \rho>0$, and $v \in \mathbb{S}^{n-1}$ we set

$$
Q_{\rho}^{v}(x):=R_{\nu} Q_{\rho}(0)+x
$$

we omit the subscript $\rho$ when $\rho=1$.

(m) For $\xi \in \mathbb{R}^{m \times n}$, the linear function from $\mathbb{R}^{n}$ to $\mathbb{R}^{m}$ with gradient $\xi$ is denoted by $\ell_{\xi}$; that is, $\ell_{\xi}(x):=\xi x$, where $x$ is considered as an $n \times 1$ matrix.

(n) For $x \in \mathbb{R}^{n}, \zeta \in \mathbb{R}_{0}^{m}$, and $v \in \mathbb{S}^{n-1}$ we define the function $u_{x, \zeta, v}$ as

$$
u_{x, \zeta, v}(y):= \begin{cases}\zeta & \text { if }(y-x) \cdot v \geq 0 \\ 0 & \text { if }(y-x) \cdot v<0\end{cases}
$$

(o) For $x \in \mathbb{R}^{n}$ and $v \in \mathbb{S}^{n-1}$, we set

$$
\Pi_{0}^{v}:=\left\{y \in \mathbb{R}^{n}: y \cdot v=0\right\} \text { and } \Pi_{x}^{v}:=\left\{y \in \mathbb{R}^{n}:(y-x) \cdot v=0\right\} .
$$

(p) For a given topological space $X, \mathscr{B}(X)$ denotes the Borel $\sigma$-algebra on $X$. In particular, for every integer $k \geq 1, \mathscr{B}^{k}$ is the Borel $\sigma$-algebra on $\mathbb{R}^{k}$, while $\mathscr{B}_{S}^{n}$ stands for the Borel $\sigma$-algebra on $\mathbb{S}^{n-1}$.

(q) For every $t \in \mathbb{R}$ the integer part of $t$ is denoted by $\lfloor t\rfloor$; that is, $\lfloor t\rfloor$ is the largest integer less than or equal to $t$.

\section{Setting of the Problem and Statements of the Main Results}

This section consists of two parts: in Section 3.1 we introduce the stochastic freediscontinuity functionals and recall the Ergodic Subadditive Theorem; in Section 3.2 we state the main results of the paper.

\subsection{Setting of the Problem}

Throughout the paper we fix six constants $p, c_{1}, \ldots, c_{5}$, with $1<p<+\infty$, $0<c_{1} \leq c_{2}<+\infty, 1 \leq c_{3}<+\infty$, and $0<c_{4} \leq c_{5}<+\infty$, and two nondecreasing continuous functions $\sigma_{1}, \sigma_{2}:[0,+\infty) \rightarrow[0,+\infty)$ such that $\sigma_{1}(0)=\sigma_{2}(0)=0$.

Definition 3.1. (Volume and surface integrands). Let $\mathcal{F}=\mathcal{F}\left(p, c_{1}, c_{2}, \sigma_{1}\right)$ be the collection of all functions $f: \mathbb{R}^{n} \times \mathbb{R}^{m \times n} \rightarrow[0,+\infty)$ satisfying the following conditions: 
( $f 1$ ) (measurability) $f$ is Borel measurable on $\mathbb{R}^{n} \times \mathbb{R}^{m \times n}$;

( $f 2$ ) (continuity in $\xi$ ) for every $x \in \mathbb{R}^{n}$ we have

$$
\left|f\left(x, \xi_{1}\right)-f\left(x, \xi_{2}\right)\right| \leq \sigma_{1}\left(\left|\xi_{1}-\xi_{2}\right|\right)\left(1+f\left(x, \xi_{1}\right)+f\left(x, \xi_{2}\right)\right)
$$

for every $\xi_{1}, \xi_{2} \in \mathbb{R}^{m \times n}$;

( $f 3$ ) (lower bound) for every $x \in \mathbb{R}^{n}$ and every $\xi \in \mathbb{R}^{m \times n}$

$$
c_{1}|\xi|^{p} \leq f(x, \xi)
$$

( $f 4$ ) (upper bound) for every $x \in \mathbb{R}^{n}$ and every $\xi \in \mathbb{R}^{m \times n}$

$$
f(x, \xi) \leq c_{2}\left(1+|\xi|^{p}\right) .
$$

Let $\mathcal{G}=\mathcal{G}\left(c_{3}, c_{4}, c_{5}, \sigma_{2}\right)$ be the collection of all functions $g: \mathbb{R}^{n} \times \mathbb{R}_{0}^{m} \times \mathbb{S}^{n-1} \rightarrow$ $[0,+\infty)$ satisfying the following conditions:

( $g 1$ ) (measurability) $g$ is Borel measurable on $\mathbb{R}^{n} \times \mathbb{R}_{0}^{m} \times \mathbb{S}^{n-1}$;

( $g 2$ ) (continuity in $\zeta$ ) for every $x \in \mathbb{R}^{n}$ and every $\nu \in \mathbb{S}^{n-1}$ we have

$$
\left|g\left(x, \zeta_{2}, v\right)-g\left(x, \zeta_{1}, v\right)\right| \leq \sigma_{2}\left(\left|\zeta_{1}-\zeta_{2}\right|\right)\left(g\left(x, \zeta_{1}, v\right)+g\left(x, \zeta_{2}, v\right)\right)
$$

for every $\zeta_{1}, \zeta_{2} \in \mathbb{R}_{0}^{m}$

(g3) (estimate for $\left|\zeta_{1}\right| \leq\left|\zeta_{2}\right|$ ) for every $x \in \mathbb{R}^{n}$ and every $v \in \mathbb{S}^{n-1}$ we have

$$
g\left(x, \zeta_{1}, v\right) \leq c_{3} g\left(x, \zeta_{2}, v\right)
$$

for every $\zeta_{1}, \zeta_{2} \in \mathbb{R}_{0}^{m}$ with $\left|\zeta_{1}\right| \leq\left|\zeta_{2}\right|$;

(g4) (estimate for $c_{3}\left|\zeta_{1}\right| \leq\left|\zeta_{2}\right|$ ) for every $x \in \mathbb{R}^{n}$ and every $v \in \mathbb{S}^{n-1}$ we have

$$
g\left(x, \zeta_{1}, v\right) \leq g\left(x, \zeta_{2}, v\right)
$$

for every $\zeta_{1}, \zeta_{2} \in \mathbb{R}_{0}^{m}$ with $c_{3}\left|\zeta_{1}\right| \leq\left|\zeta_{2}\right|$

(g5) (lower bound) for every $x \in \mathbb{R}^{n}, \zeta \in \mathbb{R}_{0}^{m}$, and $v \in \mathbb{S}^{n-1}$

$$
c_{4} \leq g(x, \zeta, v)
$$

(g6) (upper bound) for every $x \in \mathbb{R}^{n}, \zeta \in \mathbb{R}_{0}^{m}$, and $v \in \mathbb{S}^{n-1}$

$$
g(x, \zeta, v) \leq c_{5}(1+|\zeta|)
$$

(g7) (symmetry) for every $x \in \mathbb{R}^{n}, \zeta \in \mathbb{R}_{0}^{m}$, and $\nu \in \mathbb{S}^{n-1}$

$$
g(x, \zeta, v)=g(x,-\zeta,-v) .
$$

Remark 3.2. As observed in [16, Remark 3.2], assumptions ( $g 3)$ and ( $g 4)$ are strictly weaker than a monotonicity condition in $|\zeta|$. Indeed, if $g: \mathbb{R}^{n} \times \mathbb{R}_{0}^{m} \times \mathbb{S}^{n-1} \rightarrow$ $[0,+\infty)$ satisfies

$$
\zeta_{1}, \zeta_{2} \in \mathbb{R}_{0}^{m} \text { with }\left|\zeta_{1}\right| \leq\left|\zeta_{2}\right| \Longrightarrow g\left(x, \zeta_{1}, v\right) \leq g\left(x, \zeta_{2}, v\right)
$$

for every $x \in \mathbb{R}^{n}$ and every $v \in \mathbb{S}^{n-1}$, then $g$ satisfies ( $\left.g 3\right)$ and ( $\left.g 4\right)$. On the other hand, $(g 3)$ and $(g 4)$ do not imply monotonicity in $|\zeta|$. 
Given $f \in \mathcal{F}$ and $g \in \mathcal{G}$, we consider the integral functionals $F, G: L^{0}\left(\mathbb{R}^{n}, \mathbb{R}^{m}\right)$ $\times \mathscr{A} \longrightarrow[0,+\infty]$ defined as

$$
\begin{aligned}
& F(u, A):= \begin{cases}\int_{A} f(x, \nabla u) \mathrm{d} x & \text { if }\left.u\right|_{A} \in W^{1, p}\left(A, \mathbb{R}^{m}\right), \\
+\infty & \text { otherwise in } L^{0}\left(\mathbb{R}^{n}, \mathbb{R}^{m}\right) .\end{cases} \\
& G(u, A):= \begin{cases}\int_{S_{u} \cap A} g\left(x,[u], v_{u}\right) \mathrm{d} \mathcal{H}^{n-1} & \text { if }\left.u\right|_{A} \in G S B V^{p}\left(A, \mathbb{R}^{m}\right), \\
+\infty & \text { otherwise in } L^{0}\left(\mathbb{R}^{n}, \mathbb{R}^{m}\right),\end{cases}
\end{aligned}
$$

Remark 3.3. Since $[u]$ is reversed when the orientation of $v_{u}$ is reversed, the functional $G$ is well defined thanks to $(g 7)$.

Let $A \in \mathscr{A}$. For $F$ as in (3.1), and $w \in L^{0}\left(\mathbb{R}^{n}, \mathbb{R}^{m}\right)$ with $\left.w\right|_{A} \in W^{1, p}\left(A, \mathbb{R}^{m}\right)$, we set

$m_{F}^{1, p}(w, A):=\inf \left\{F(u, A): u \in L^{0}\left(\mathbb{R}^{n}, \mathbb{R}^{m}\right),\left.u\right|_{A} \in W^{1, p}\left(A, \mathbb{R}^{m}\right), u=w\right.$ near $\left.\partial A\right\}$.

Moreover, for $G$ as in (3.2), and $w \in L^{0}\left(\mathbb{R}^{n}, \mathbb{R}^{m}\right)$ with $\left.w\right|_{A} \in S B V_{\mathrm{pc}}\left(A, \mathbb{R}^{m}\right)$, we set

$$
m_{G}^{\mathrm{pc}}(w, A):=\inf \left\{G(u, A): u \in L^{0}\left(\mathbb{R}^{n}, \mathbb{R}^{m}\right),\left.u\right|_{A} \in S B V_{\mathrm{pc}}\left(A, \mathbb{R}^{m}\right), u=w \text { near } \partial A\right\}
$$

In (3.3) and (3.4), by " $u=w$ near $\partial A$ " we mean that there exists a neighbourhood $U$ of $\partial A$ such that $u=w \mathcal{L}^{n}$-almost everywhere in $U$.

If $A$ is an arbitrary bounded subset of $\mathbb{R}^{n}$, we set $m_{F}^{1, p}(w, A):=m_{F}^{1, p}(w, \operatorname{int} A)$ and $m_{G}^{\mathrm{pc}}(w, A):=m_{G}^{\mathrm{pc}}(w, \operatorname{int} A)$, where int denotes the interior of $A$.

Remark 3.4. Let $u \in L^{0}\left(\mathbb{R}^{n}, \mathbb{R}^{m}\right)$ be such that $\left.u\right|_{A} \in S B V_{\mathrm{pc}}\left(A, \mathbb{R}^{m}\right)$, and let $k \in \mathbb{N}$. A careful inspection of the proof of [16, Lemma 4.1] shows that there exist $\mu_{k}>k$ and $v_{k} \in L^{\infty}\left(\mathbb{R}^{n}, \mathbb{R}^{m}\right)$ with $\left.v_{k}\right|_{A} \in S B V_{\mathrm{pc}}\left(A, \mathbb{R}^{m}\right)$ such that

$$
\begin{aligned}
\left\|v_{k}\right\|_{L^{\infty}\left(A, \mathbb{R}^{m}\right)} & \leq \mu_{k}, \quad v_{k}=u \mathcal{L}^{n} \text {-almost everywhere in }\{|u| \leq k\} \\
G\left(v_{k}, A\right) & \leq\left(1+\frac{1}{k}\right) G(u, A)
\end{aligned}
$$

As a consequence we may readily deduce the following. Let $w \in L^{0}\left(\mathbb{R}^{n}, \mathbb{R}^{m}\right)$ be such that $\left.w\right|_{A} \in S B V_{\mathrm{pc}}\left(A, \mathbb{R}^{m}\right) \cap L^{\infty}\left(A, \mathbb{R}^{m}\right)$ and let $k \in \mathbb{N}, k>\|w\|_{L^{\infty}\left(A, \mathbb{R}^{m}\right)}$ be fixed. Then

$$
m_{G}^{\mathrm{pc}}(w, A)=\inf _{k} m_{G}^{k}(w, A)=\lim _{k \rightarrow+\infty} m_{G}^{k}(w, A),
$$

where

$$
\begin{array}{r}
m_{G}^{k}(w, A):=\inf \left\{G(u, A): u \in L^{0}\left(\mathbb{R}^{n}, \mathbb{R}^{m}\right),\left.u\right|_{A} \in S B V_{\mathrm{pc}}\left(A, \mathbb{R}^{m}\right) \cap L^{\infty}\left(A, \mathbb{R}^{m}\right),\right. \\
\left.\|u\|_{L^{\infty}\left(A, \mathbb{R}^{m}\right)} \leq k, \mathcal{H}^{n-1}\left(S_{u} \cap A\right) \leq \alpha, u=w \text { near } \partial A\right\},
\end{array}
$$


with $\alpha:=c_{5} / c_{4}\left(1+2\|w\|_{L^{\infty}\left(A, \mathbb{R}^{m}\right)}\right) \mathcal{H}^{n-1}\left(S_{w} \cap A\right)$. The fact that the inequality $\mathcal{H}^{n-1}\left(S_{u} \cap A\right) \leq \alpha$ in (3.6) is not restrictive follows from assumption ( $g 6$ ) by using $w$ as a competitor in the minimisation problem defining $m_{G}^{k}(w, A)$ for $k>$ $\|w\|_{L^{\infty}\left(A, \mathbb{R}^{m}\right)}$.

We are now ready to introduce the probabilistic setting of our problem. In what follows $(\Omega, \mathcal{T}, P)$ denotes a fixed probability space.

Definition 3.5. (Random integrand). A function $f: \Omega \times \mathbb{R}^{n} \times \mathbb{R}^{m \times n} \rightarrow[0,+\infty)$ is called a random volume integrand if

(a) $f$ is $\left(\mathcal{T} \otimes \mathscr{B}^{n} \otimes \mathscr{B}^{m \times n}\right)$-measurable;

(b) $f(\omega, \cdot, \cdot) \in \mathcal{F}$ for every $\omega \in \Omega$.

A function $g: \Omega \times \mathbb{R}^{n} \times \mathbb{R}_{0}^{m} \times \mathbb{S}^{n-1} \rightarrow[0,+\infty)$ is called a random surface integrand if

(c) $g$ is $\left(\mathcal{T} \otimes \mathscr{B}^{n} \otimes \mathscr{B}^{m} \otimes \mathscr{B}_{S}^{n}\right)$-measurable;

(d) $g(\omega, \cdot, \cdot, \cdot) \in \mathcal{G}$ for every $\omega \in \Omega$.

Let $f$ be a random volume integrand. For $\omega \in \Omega$ the integral functional $F(\omega): L^{0}\left(\mathbb{R}^{n}, \mathbb{R}^{m}\right) \times \mathscr{A} \longrightarrow[0,+\infty]$ is defined by $(3.1)$, with $f(\cdot, \cdot)$ replaced by $f(\omega, \cdot, \cdot)$. Let $g$ be a random surface integrand. For $\omega \in \Omega$ the integral functional $G(\omega): L^{0}\left(\mathbb{R}^{n}, \mathbb{R}^{m}\right) \times \mathscr{A} \longrightarrow[0,+\infty]$ is defined by $(3.2)$, with $g(\cdot, \cdot, \cdot)$ replaced by $g(\omega, \cdot, \cdot, \cdot)$. Finally, for every $\varepsilon>0$ we consider the free-discontinuity functional $E_{\varepsilon}(\omega): L^{0}\left(\mathbb{R}^{n}, \mathbb{R}^{m}\right) \times \mathscr{A} \longrightarrow[0,+\infty]$ defined by

$$
\begin{aligned}
& E_{\varepsilon}(\omega)(u, A):= \\
& \begin{cases}\int_{A} f\left(\omega, \frac{x}{\varepsilon}, \nabla u\right) \mathrm{d} x+\int_{S_{u} \cap A} g\left(\omega, \frac{x}{\varepsilon},[u], v_{u}\right) \mathrm{d} \mathcal{H}^{n-1} & \text { if }\left.u\right|_{A} \in G S B V^{p}\left(A, \mathbb{R}^{m}\right), \\
+\infty & \text { otherwise in } L^{0}\left(\mathbb{R}^{n}, \mathbb{R}^{m}\right) .\end{cases}
\end{aligned}
$$

In the study of stochastic homogenisation an important role is played by the notions introduced by the following definitions:

Definition 3.6. (P-preserving transformation). A $P$-preserving transformation on $(\Omega, \mathcal{T}, P)$ is a map $T: \Omega \rightarrow \Omega$ satisfying the following properties:

(a) (measurability) $T$ is $\mathcal{T}$-measurable;

(b) (bijectivity) $T$ is bijective;

(c) (invariance) $P(T(E))=P(E)$, for every $E \in \mathcal{T}$.

If, in addition, every set $E \in \mathcal{T}$ which satisfies $P(T(E) \triangle E)=0$ (called $T$ invariant set according to [24]) has probability 0 or 1 , then $T$ is called ergodic. Here and henceforth $\triangle$ denotes the symmetric difference of sets.

Definition 3.7. (Group of P-preserving transformations). Let $k$ be a positive integer. A group of $P$-preserving transformations on $(\Omega, \mathcal{T}, P)$ is a family $\left(\tau_{z}\right)_{z \in \mathbb{Z}^{k}}$ (resp. $\left(\tau_{z}\right)_{z \in \mathbb{R}^{k}}$ ) of mappings $\tau_{z}: \Omega \rightarrow \Omega$ satisfying the following properties: 
(a) (measurability) $\tau_{z}$ is $\mathcal{T}$-measurable for every $z \in \mathbb{Z}^{k}$ (resp. $z \in \mathbb{R}^{k}$ );

(b) (bijectivity) $\tau_{z}$ is bijective for every $z \in \mathbb{Z}^{k}$ (resp. $z \in \mathbb{R}^{k}$ );

(c) (invariance) $P\left(\tau_{z}(E)\right)=P(E)$, for every $E \in \mathcal{T}$ and every $z \in \mathbb{Z}^{k}$ (resp. $\left.z \in \mathbb{R}^{k}\right)$

(d) (group property) $\tau_{0}=\mathrm{i} d_{\Omega}$ (the identity map on $\Omega$ ) and $\tau_{z+z^{\prime}}=\tau_{z} \circ \tau_{z^{\prime}}$ for every $z, z^{\prime} \in \mathbb{Z}^{k}$ (resp. $z, z^{\prime} \in \mathbb{R}^{k}$ ).

If, in addition, every set $E \in \mathcal{T}$ which satisfies $P\left(\tau_{z}(E) \triangle E\right)=0$ for every $z \in \mathbb{Z}^{k}$ (resp. $z \in \mathbb{R}^{k}$ ) has probability 0 or 1 , then $\left(\tau_{z}\right)_{z \in \mathbb{Z}^{k}}\left(\right.$ resp. $\left.\left(\tau_{z}\right)_{z \in \mathbb{R}^{k}}\right)$ is called ergodic.

Remark 3.8. In the case $k=1$ a group of $P$-preserving transformations $\left(\tau_{z}\right)_{z \in \mathbb{Z}}$ has the form $\left(T^{z}\right)_{z \in \mathbb{Z}}$, where $T:=\tau_{1}$ is a $P$-preserving transformation.

We are now in a position to define the notion of stationary random integrand.

Definition 3.9. (Stationary random integrand). A random volume integrand $f$ is stationary with respect to a group $\left(\tau_{z}\right)_{z \in \mathbb{Z}^{n}}\left(\operatorname{resp} .\left(\tau_{z}\right)_{z \in \mathbb{R}^{n}}\right)$ of $P$-preserving transformations on $(\Omega, \mathcal{T}, P)$ if

$$
f(\omega, x+z, \xi)=f\left(\tau_{z}(\omega), x, \xi\right)
$$

for every $\omega \in \Omega, x \in \mathbb{R}^{n}, z \in \mathbb{Z}^{n}$ (resp. $z \in \mathbb{R}^{n}$ ), and $\xi \in \mathbb{R}^{m \times n}$.

Similarly, a random surface integrand $g$ is stationary with respect to $\left(\tau_{z}\right)_{z \in \mathbb{Z}^{n}}$ (resp. $\left.\left(\tau_{z}\right)_{z \in \mathbb{R}^{n}}\right)$ if

$$
g(\omega, x+z, \zeta, v)=g\left(\tau_{z}(\omega), x, \zeta, v\right)
$$

for every $\omega \in \Omega, x \in \mathbb{R}^{n}, z \in \mathbb{Z}^{n}$ (resp. $z \in \mathbb{R}^{n}$ ), $\zeta \in \mathbb{R}_{0}^{m}$, and $v \in \mathbb{S}^{n-1}$.

We now recall the notion of subadditive stochastic processes as well as the Subadditive Ergodic Theorem by AKcoglu and Krengel [1, Theorem 2.7].

Let $k$ be a positive integer. For every $a, b \in \mathbb{R}^{k}$, with $a_{i}<b_{i}$ for $i=1, \ldots, k$, we define

$$
[a, b):=\left\{x \in \mathbb{R}^{k}: a_{i} \leq x_{i}<b_{i} \text { for } i=1, \ldots, k\right\}
$$

and we set

$$
\mathcal{I}_{k}:=\left\{[a, b): a, b \in \mathbb{R}^{k}, a_{i}<b_{i} \text { for } i=1, \ldots, k\right\} .
$$

Definition 3.10. (Subadditive process). A subadditive process with respect to a group $\left(\tau_{z}\right)_{z \in \mathbb{Z}^{k}}\left(\operatorname{resp} .\left(\tau_{z}\right)_{z \in \mathbb{R}^{k}}\right), k \geq 1$, of $P$-preserving transformations on $(\Omega, \mathcal{T}, P)$ is a function $\mu: \Omega \times \mathcal{I}_{k} \rightarrow \mathbb{R}$ satisfying the following properties:

(a) (measurability) for every $A \in \mathcal{I}_{k}$ the function $\omega \mapsto \mu(\omega, A)$ is $\mathcal{T}$-measurable;

(b) (covariance) for every $\omega \in \Omega, A \in \mathcal{I}_{k}$, and $z \in \mathbb{Z}^{k}$ (resp. $z \in \mathbb{R}^{k}$ ) we have $\mu(\omega, A+z)=\mu\left(\tau_{z}(\omega), A\right) ;$

(c) (subadditivity) for every $A \in \mathcal{I}_{k}$ and for every finite family $\left(A_{i}\right)_{i \in I} \subset \mathcal{I}_{k}$ of pairwise disjoint sets such that $A=\cup_{i \in I} A_{i}$, we have

$$
\mu(\omega, A) \leq \sum_{i \in I} \mu\left(\omega, A_{i}\right) \text { for every } \omega \in \Omega
$$


(d) (boundedness) there exists $c>0$ such that $0 \leq \mu(\omega, A) \leq c \mathcal{L}^{k}(A)$ for every $\omega \in \Omega$ and every $A \in \mathcal{I}_{k}$.

We now state a variant of the Pointwise Ergodic Theorem which is suitable for our purposes.

Theorem 3.11. (Subadditive Ergodic Theorem). Let $k$ be a positive integer and let $\left(\tau_{z}\right)_{z \in \mathbb{Z}^{k}}\left(\right.$ resp. $\left.\left(\tau_{z}\right)_{z \in \mathbb{R}^{k}}\right)$ be a group of $P$-preserving transformations on $(\Omega, \mathcal{T}, P)$. Let $\mu: \Omega \times \mathcal{I}_{k} \rightarrow \mathbb{R}$ be a subadditive process with respect to $\left(\tau_{z}\right)_{z \in \mathbb{Z}^{k}}$ (resp. $\left.\left(\tau_{z}\right)_{z \in \mathbb{R}^{k}}\right)$. Then there exist a $\mathcal{T}$-measurable function $\varphi: \Omega \rightarrow[0,+\infty)$ and a set $\Omega^{\prime} \in \mathcal{T}$ with $P\left(\Omega^{\prime}\right)=1$ such that

$$
\lim _{t \rightarrow+\infty} \frac{\mu(\omega, t Q)}{\mathcal{L}^{k}(t Q)}=\varphi(\omega)
$$

for every $\omega \in \Omega^{\prime}$ and for every cube $Q \in \mathcal{I}_{k}$. If in addition $\left(\tau_{z}\right)_{z \in \mathbb{Z}^{k}}\left(\right.$ resp. $\left.\left(\tau_{z}\right)_{z \in \mathbb{R}^{k}}\right)$ is ergodic, then $\varphi$ is constant $P$-almost everywhere

Proof. If the set of indices is $\mathbb{Z}^{k}$, the proof can be found in [1, Theorem 2.7 and Remark p. 59] (see, for example, [18, Proposition 1]). If the set of indices is $\mathbb{R}^{k}$, the existence of $\varphi$ and $\Omega^{\prime}$ satisfying (3.10) can be proved by considering the restriction of the group to $\mathbb{Z}^{k}$. Since ergodicity for $\left(\tau_{z}\right)_{z \in \mathbb{R}^{k}}$ does not imply ergodicity for $\left(\tau_{z}\right)_{z \in \mathbb{Z}^{k}}$, we have to prove the last sentence of the theorem when the set of indices is $\mathbb{R}^{k}$.

Let $\mathcal{Q}$ be the set of all cubes $Q \in \mathcal{I}_{k}$ with vertices in $\mathbb{Q}^{k}$, let $\Omega^{\prime \prime}$ be the set of all $\omega \in \Omega$ such that the limit

$$
\lim _{\substack{t \rightarrow+\infty \\ t \in \mathbb{Q}}} \frac{\mu(\omega, t Q)}{\mathcal{L}^{k}(t Q)}
$$

exists for every $Q \in \mathcal{Q}$, and let $\widehat{\Omega}$ be the set of all $\omega \in \Omega^{\prime \prime}$ such that the above limits do not depend on $Q \in \mathcal{Q}$. Since $\omega \mapsto \mu(\omega, Q)$ is $\mathcal{T}$-measurable, we have $\Omega^{\prime \prime} \in \mathcal{T}$ and the limit in (3.11) is a $\mathcal{T}$-measurable function of $\omega$. This implies that $\widehat{\Omega} \in \mathcal{T}$ and that there exists a $\mathcal{T}$-measurable function $\hat{\varphi}: \widehat{\Omega} \rightarrow \mathbb{R}$ such that

$$
\lim _{\substack{t \rightarrow+\infty \\ t \in \mathbb{Q}}} \frac{\mu(\omega, t Q)}{\mathcal{L}^{k}(t Q)}=\hat{\varphi}(\omega)
$$

for every $\omega \in \widehat{\Omega}$ and $Q \in \mathcal{Q}$. By (3.10) we have

$$
P(\widehat{\Omega})=1 \text { and } \hat{\varphi}(\omega)=\varphi(\omega) \text { for } P \text {-almost everywhere } \omega \in \widehat{\Omega} \text {. }
$$

Fix $\omega \in \widehat{\Omega}, z \in \mathbb{R}^{k}$, and $Q \in \mathcal{Q}$. By covariance (condition (b) of Definition 3.10) we have

$$
\mu\left(\tau_{z}(\omega), t Q\right)=\mu\left(\omega, t\left(Q+\frac{z}{t}\right)\right)
$$

for every $t>0$. Given $Q^{\prime}, Q^{\prime \prime} \in \mathcal{Q}$, with $Q^{\prime} \subset \subset$ int $Q \subset \bar{Q} \subset \subset Q^{\prime \prime}$, for $t$ large enough we have

$$
Q^{\prime} \subset Q+\frac{z}{t} \text { and } Q+\frac{z}{t} \subset Q^{\prime \prime}
$$


By subadditivity and boundedness (conditions (c) and (d) of Definition 3.10) we have

$$
\begin{aligned}
& \mu\left(\omega, t\left(Q+\frac{z}{t}\right)\right) \leq \mu\left(\omega, t Q^{\prime}\right)+c t^{k} \mathcal{L}^{k}\left(\left(Q+\frac{z}{t}\right) \backslash Q^{\prime}\right), \\
& \mu\left(\omega, t Q^{\prime \prime}\right) \leq \mu\left(\omega, t\left(Q+\frac{z}{t}\right)\right)+c t^{k} \mathcal{L}^{k}\left(Q^{\prime \prime} \backslash\left(Q+\frac{z}{t}\right)\right),
\end{aligned}
$$

hence

$$
\begin{aligned}
& \frac{\mu\left(\omega, t\left(Q+\frac{z}{t}\right)\right)}{\mathcal{L}^{k}(t Q)} \leq \frac{\mu\left(\omega, t Q^{\prime}\right)}{\mathcal{L}^{k}\left(t Q^{\prime}\right)}+c \frac{\mathcal{L}^{k}\left(\left(Q+\frac{z}{t}\right) \backslash Q^{\prime}\right)}{\mathcal{L}^{k}(Q)}, \\
& \frac{\mu\left(\omega, t Q^{\prime \prime}\right)}{\mathcal{L}^{k}\left(t Q^{\prime \prime}\right)} \leq \frac{\mu\left(\omega, t\left(Q+\frac{z}{t}\right)\right)}{\mathcal{L}^{k}(t Q)}+c \frac{\mathcal{L}^{k}\left(Q^{\prime \prime} \backslash\left(Q+\frac{z}{t}\right)\right)}{\mathcal{L}^{k}(Q)}
\end{aligned}
$$

Therefore by (3.12) and (3.14) we obtain

$$
\begin{aligned}
& \limsup _{\substack{t \rightarrow+\infty \\
t \in \mathbb{Q}}} \frac{\mu\left(\tau_{z}(\omega), t Q\right)}{\mathcal{L}^{k}(t Q)} \leq \hat{\varphi}(\omega)+c \frac{\mathcal{L}^{k}\left(Q \backslash Q^{\prime}\right)}{\mathcal{L}^{k}(Q)}, \\
& \hat{\varphi}(\omega) \leq \liminf _{\substack{t \rightarrow+\infty \\
t \in \mathbb{Q}}} \frac{\mu\left(\tau_{z}(\omega), t Q\right)}{\mathcal{L}^{k}(t Q)}+c \frac{\mathcal{L}^{k}\left(Q^{\prime \prime} \backslash Q\right)}{\mathcal{L}^{k}(Q)} .
\end{aligned}
$$

Taking the limit as $Q^{\prime} \nearrow Q$ and $Q^{\prime \prime} \searrow Q$ we obtain

$$
\hat{\varphi}(\omega) \leq \liminf _{\substack{t \rightarrow+\infty \\ t \in \mathbb{Q}}} \frac{\mu\left(\tau_{z}(\omega), t Q\right)}{\mathcal{L}^{k}(t Q)} \leq \limsup _{\substack{t \rightarrow+\infty \\ t \in \mathbb{Q}}} \frac{\mu\left(\tau_{z}(\omega), t Q\right)}{\mathcal{L}^{k}(t Q)} \leq \hat{\varphi}(\omega),
$$

which implies that $\tau_{z}(\omega) \in \Omega^{\prime \prime}$ and

$$
\lim _{\substack{t \rightarrow+\infty \\ t \in \mathbb{Q}}} \frac{\mu\left(\tau_{z}(\omega), t Q\right)}{\mathcal{L}^{k}(t Q)}=\hat{\varphi}(\omega) .
$$

Since the limit does not depend on $Q \in \mathcal{Q}$ we have also $\tau_{z}(\omega) \in \widehat{\Omega}$. By (3.12) and (3.15) we have $\hat{\varphi}\left(\tau_{z}(\omega)\right)=\hat{\varphi}(\omega)$ for every $\omega \in \widehat{\Omega}$ and every $z \in \mathbb{R}^{k}$. This implies that, for every $c \in \mathbb{R}$, the superlevel sets of $\hat{\varphi}$,

$$
E_{c}:=\{\omega \in \widehat{\Omega}: \hat{\varphi}(\omega) \geq c\},
$$

are invariant for $\tau_{z}$ for every $z \in \mathbb{R}^{k}$. Therefore, if $\left(\tau_{z}\right)_{z \in \mathbb{R}^{k}}$ is ergodic, we can only have

$$
P\left(E_{c}\right)=0 \text { or } P\left(E_{c}\right)=1 \text {. }
$$

Since $E_{c_{1}} \supset E_{c_{2}}$ for $c_{1}<c_{2}$, by (3.16) there exists $c_{0} \in \mathbb{R}$ such that $P\left(E_{c}\right)=0$ for $c>c_{0}$ and $P\left(E_{c}\right)=1$ for $c<c_{0}$. It then follows that $\hat{\varphi}$ is constant $P$-almost everywhere, and so is $\varphi$ by (3.13). 


\subsection{Statement of the Main Results}

In this section we state the main result of the paper, Theorem 3.13, which provides a $\Gamma$-convergence and integral representation result for the random functionals $\left(E_{\varepsilon}(\omega)\right)_{\varepsilon>0}$ introduced in (3.7), under the assumption that the volume and surface integrands $f$ and $g$ are stationary. The volume and surface integrands of the $\Gamma$-limit are given in terms of separate asymptotic cell formulas, showing that there is no interaction between volume and surface densities by stochastic $\Gamma$-convergence.

The next theorem proves the existence of the limits in the asymptotic cell formulas that will be used in the statement of the main result. The proof will be given in Sections 4-6.

Theorem 3.12. (Homogenisation formulas). Let $f$ be a stationary random volume integrand and let $g$ be a stationary random surface integrand with respect to a group $\left(\tau_{z}\right)_{z \in \mathbb{Z}^{n}}$ (resp. $\left.\left(\tau_{z}\right)_{z \in \mathbb{R}^{n}}\right)$ of P-preserving transformations on $(\Omega, \mathcal{T}, P)$. For every $\omega \in \Omega$ let $F(\omega)$ and $G(\omega)$ be defined by (3.1) and (3.2), with $f(\cdot, \cdot)$ and $g(\cdot, \cdot, \cdot)$ replaced by $f(\omega, \cdot, \cdot)$ and $g(\omega, \cdot, \cdot, \cdot)$, respectively. Finally, let $m_{F(\omega)}^{1, p}$ and $m_{G(\omega)}^{\mathrm{pc}}$ be defined by (3.3) and (3.4), respectively. Then there exists $\Omega^{\prime} \in \mathcal{T}$, with $P\left(\Omega^{\prime}\right)=1$, such that for every $\omega \in \Omega^{\prime}, x \in \mathbb{R}^{n}, \xi \in \mathbb{R}^{m \times n}, \zeta \in \mathbb{R}_{0}^{m}$, and $v \in \mathbb{S}^{n-1}$ the limits

$$
\lim _{t \rightarrow+\infty} \frac{m_{F(\omega)}^{1, p}\left(\ell_{\xi}, Q_{t}(t x)\right)}{t^{n}} \text { and } \lim _{t \rightarrow+\infty} \frac{m_{G(\omega)}^{\mathrm{pc}}\left(u_{t x, \zeta, \nu}, Q_{t}^{v}(t x)\right)}{t^{n-1}}
$$

exist and are independent of $x$. More precisely, there exist a random volume integrand $f_{\text {hom }}: \Omega \times \mathbb{R}^{m \times n} \rightarrow[0,+\infty)$, and a random surface integrand $g_{\mathrm{hom}}: \Omega \times$ $\mathbb{R}_{0}^{m} \times \mathbb{S}^{n-1} \rightarrow[0,+\infty)$ such that for every $\omega \in \Omega^{\prime}, x \in \mathbb{R}^{n}, \xi \in \mathbb{R}^{m \times n}, \zeta \in \mathbb{R}_{0}^{m}$, and $v \in \mathbb{S}^{n-1}$

$$
\begin{aligned}
f_{\text {hom }}(\omega, \xi) & =\lim _{t \rightarrow+\infty} \frac{m_{F(\omega)}^{1, p}\left(\ell_{\xi}, Q_{t}(t x)\right)}{t^{n}}=\lim _{t \rightarrow+\infty} \frac{m_{F(\omega)}^{1, p}\left(\ell_{\xi}, Q_{t}(0)\right)}{t^{n}} \\
g_{\text {hom }}(\omega, \zeta, v) & =\lim _{t \rightarrow+\infty} \frac{m_{G(\omega)}^{\mathrm{pc}}\left(u_{t x, \zeta, v}, Q_{t}^{v}(t x)\right)}{t^{n-1}}=\lim _{t \rightarrow+\infty} \frac{m_{G(\omega)}^{\mathrm{pc}}\left(u_{0, \zeta, v}, Q_{t}^{\nu}(0)\right)}{t^{n-1}} .
\end{aligned}
$$

If, in addition, $\left(\tau_{z}\right)_{z \in \mathbb{Z}^{n}}$ (resp. $\left.\left(\tau_{z}\right)_{z \in \mathbb{R}^{n}}\right)$ is ergodic, then $f_{\text {hom }}$ and $g_{\text {hom }}$ are independent of $\omega$ and

$$
\begin{aligned}
f_{\text {hom }}(\xi) & =\lim _{t \rightarrow+\infty} \frac{1}{t^{n}} \int_{\Omega} m_{F(\omega)}^{1, p}\left(\ell_{\xi}, Q_{t}(0)\right) \mathrm{d} P(\omega), \\
g_{\text {hom }}(\zeta, v) & =\lim _{t \rightarrow+\infty} \frac{1}{t^{n-1}} \int_{\Omega} m_{G(\omega)}^{\mathrm{pc}}\left(u_{0, \zeta, v}, Q_{t}^{v}(0)\right) \mathrm{d} P(\omega) .
\end{aligned}
$$

We are now ready to state the main result of this paper, namely the almost sure $\Gamma$-convergence of the sequence of random functionals $\left(E_{\varepsilon}(\omega)\right)_{\varepsilon>0}$ introduced in (3.7). 
Theorem 3.13. ( $\Gamma$-convergence). Let $f$ and $g$ be stationary random volume and surface integrands with respect to a group $\left(\tau_{z}\right)_{z \in \mathbb{Z}^{n}}$ (resp. $\left(\tau_{z}\right)_{z \in \mathbb{R}^{n}}$ ) of P-preserving transformations on $(\Omega, \mathcal{T}, P)$, let $E_{\varepsilon}(\omega)$ be as in (3.7), let $\Omega^{\prime} \in \mathcal{T}$ (with $P\left(\Omega^{\prime}\right)=$ $1), f_{\mathrm{hom}}$, and $g_{\mathrm{hom}}$ be as in Theorem 3.12 , and let $E_{\mathrm{hom}}(\omega): L^{0}\left(\mathbb{R}^{n}, \mathbb{R}^{m}\right) \times \mathscr{A} \longrightarrow$ $[0,+\infty]$ be the free-discontinuity functional defined by

$$
\begin{aligned}
& E_{\mathrm{hom}}(\omega)(u, A) \\
& = \begin{cases}\int_{A} f_{\mathrm{hom}}(\omega, \nabla u) \mathrm{d} x+\int_{S_{u} \cap A} g_{\mathrm{hom}}\left(\omega,[u], v_{u}\right) \mathrm{d} \mathcal{H}^{n-1} & \text { if }\left.u\right|_{A} \in G S B V^{p}\left(A, \mathbb{R}^{m}\right), \\
+\infty & \text { otherwise in } L^{0}\left(\mathbb{R}^{n}, \mathbb{R}^{m}\right) .\end{cases}
\end{aligned}
$$

Let moreover $E_{\varepsilon}^{p}(\omega)$ and $E_{\mathrm{hom}}^{p}(\omega)$ be the restrictions to $L_{\mathrm{loc}}^{p}\left(\mathbb{R}^{n}, \mathbb{R}^{m}\right) \times \mathscr{A}$ of $E_{\varepsilon}(\omega)$ and $E_{\mathrm{hom}}(\omega)$, respectively. Then

$$
E_{\varepsilon}(\omega)(\cdot, A) \Gamma \text {-converge to } E_{\mathrm{hom}}(\omega)(\cdot, A) \text { in } L^{0}\left(\mathbb{R}^{n}, \mathbb{R}^{m}\right),
$$

and

$$
E_{\varepsilon}^{p}(\omega)(\cdot, A) \Gamma \text {-converge to } E_{\mathrm{hom}}^{p}(\omega)(\cdot, A) \text { in } L_{\mathrm{loc}}^{p}\left(\mathbb{R}^{n}, \mathbb{R}^{m}\right),
$$

for every $\omega \in \Omega^{\prime}$ and every $A \in \mathscr{A}$.

Further, if $\left(\tau_{z}\right)_{z \in \mathbb{Z}^{n}}$ (resp. $\left.\left(\tau_{z}\right)_{z \in \mathbb{R}^{n}}\right)$ is ergodic, then $E_{\mathrm{hom}}$ (resp. $E_{\mathrm{hom}}^{p}$ ) is a deterministic functional; that is, it does not depend on $\omega$.

Proof. Let $\Omega^{\prime} \in \mathcal{T}$ be the set with $P\left(\Omega^{\prime}\right)=1$ whose existence is established in Theorem 3.12 and let $\omega \in \Omega^{\prime}$ be fixed. Then, the functionals $F(\omega)$ and $G(\omega)$ defined by (3.1) and (3.2), respectively (with $f(\cdot, \cdot)$ replaced by $f(\omega, \cdot, \cdot)$ and $g(\cdot, \cdot, \cdot)$ replaced by $g(\omega, \cdot, \cdot, \cdot))$ satisfy all the assumptions of [16, Theorem 3.8]. Therefore, by combining Theorem 3.12 and [16, Theorem 3.8] the conclusion follows.

Thanks to Theorem 3.13 we can also characterise the asymptotic behaviour of some minimisation problems involving $E_{\varepsilon}(\omega)$. An example is shown in the corollary below.

Corollary 3.14. (Convergence of minimisation problems). Let $f$ and $g$ be stationary random volume and surface integrands with respect to a group $\left(\tau_{z}\right)_{z \in \mathbb{Z}^{n}}$ (resp. $\left.\left(\tau_{z}\right)_{z \in \mathbb{R}^{n}}\right)$ of P-preserving transformations on $(\Omega, \mathcal{T}, P)$, let $\Omega^{\prime} \in \mathcal{T}$ (with $\left.P\left(\Omega^{\prime}\right)=1\right)$, fhom, and $g_{\text {hom }}$ be as in Theorem 3.12. Let $\omega \in \Omega^{\prime}, A \in \mathscr{A}$, $h \in L^{p}\left(A, \mathbb{R}^{m}\right)$, and let $\left(u_{\varepsilon}\right)_{\varepsilon>0} \subset G S B V^{p}\left(A, \mathbb{R}^{m}\right) \cap L^{p}\left(A, \mathbb{R}^{m}\right)$ be a sequence such that

$$
\begin{aligned}
& \int_{A} f\left(\omega, \frac{x}{\varepsilon}, \nabla u_{\varepsilon}\right) \mathrm{d} x+\int_{S_{u_{\varepsilon}} \cap A} g\left(\omega, \frac{x}{\varepsilon},\left[u_{\varepsilon}\right], v_{u_{\varepsilon}}\right) \mathrm{d} \mathcal{H}^{n-1}+\int_{A}\left|u_{\varepsilon}-h\right|^{p} \mathrm{~d} x \\
& \leq \inf _{u}\left(\int_{A} f\left(\omega, \frac{x}{\varepsilon}, \nabla u\right) \mathrm{d} x+\int_{S_{u} \cap A} g\left(\omega, \frac{x}{\varepsilon},[u], v_{u}\right) \mathrm{d} \mathcal{H}^{n-1}+\int_{A}|u-h|^{p} \mathrm{~d} x\right)+\eta_{\varepsilon}
\end{aligned}
$$

for some $\eta_{\varepsilon} \rightarrow 0+$, where the infimum is taken over all $u \in G S B V^{p}\left(A, \mathbb{R}^{m}\right) \cap$ $L^{p}\left(A, \mathbb{R}^{m}\right)$. Then there exists a sequence $\varepsilon_{k} \rightarrow 0+$ such that $\left(u_{\varepsilon_{k}}\right)_{k \in \mathbb{N}}$ converges in $L^{p}\left(A, \mathbb{R}^{m}\right)$ to a minimiser $u_{0}$ of

$$
\int_{A} f_{\text {hom }}(\omega, \nabla u) \mathrm{d} x+\int_{S_{u} \cap A} g_{\text {hom }}\left(\omega,[u], v_{u}\right) \mathrm{d} \mathcal{H}^{n-1}+\int_{A}|u-h|^{p} \mathrm{~d} x
$$


on $G S B V^{p}\left(A, \mathbb{R}^{m}\right) \cap L^{p}\left(A, \mathbb{R}^{m}\right)$. Moreover

$$
\int_{A} f\left(\omega, \frac{x}{\varepsilon}, \nabla u_{\varepsilon}\right) \mathrm{d} x+\int_{S_{u_{\varepsilon}} \cap A} g\left(\omega, \frac{x}{\varepsilon},\left[u_{\varepsilon}\right], v_{u_{\varepsilon}}\right) \mathrm{d} \mathcal{H}^{n-1}+\int_{A}\left|u_{\varepsilon}-h\right|^{p} \mathrm{~d} x
$$

converges to

$$
\int_{A} f_{\text {hom }}\left(\omega, \nabla u_{0}\right) \mathrm{d} x+\int_{S_{u_{0}} \cap A} g_{\text {hom }}\left(\omega,\left[u_{0}\right], v_{u_{0}}\right) \mathrm{d} \mathcal{H}^{n-1}+\int_{A}\left|u_{0}-h\right|^{p} \mathrm{~d} x
$$

as $\varepsilon \rightarrow 0+$.

Proof. The proof follows from Theorem 3.13, arguing as in the proof of [16, Corollary 6.1].

\section{Proof of the Cell-Formula for the Volume Integrand}

In this section we prove (3.17).

Proposition 4.1. (Homogenised volume integrand). Let $f$ be a stationary random volume integrand with respect to a group $\left(\tau_{z}\right)_{z \in \mathbb{Z}^{n}}$ (resp. $\left.\left(\tau_{z}\right)_{z \in \mathbb{R}^{n}}\right)$ of P-preserving transformations on $(\Omega, \mathcal{T}, P)$. Then there exists $\Omega^{\prime} \in \mathcal{T}$, with $P\left(\Omega^{\prime}\right)=1$, such that for every $\omega \in \Omega^{\prime}$, for every $x \in \mathbb{R}^{n}$, and $\xi \in \mathbb{R}^{m \times n}$ the limit

$$
\lim _{t \rightarrow+\infty} \frac{m_{F(\omega)}^{1, p}\left(\ell_{\xi}, Q_{t}(t x)\right)}{t^{n}}
$$

exists and is independent of $x$. More precisely, there exists a random volume integrand $f_{\mathrm{hom}}: \Omega \times \mathbb{R}^{m \times n} \rightarrow[0,+\infty)$, independent of $x$, such that

$$
f_{\mathrm{hom}}(\omega, \xi)=\lim _{t \rightarrow+\infty} \frac{m_{F(\omega)}^{1, p}\left(\ell_{\xi}, Q_{t}(t x)\right)}{t^{n}}=\lim _{t \rightarrow+\infty} \frac{m_{F(\omega)}^{1, p}\left(\ell_{\xi}, Q_{t}(0)\right)}{t^{n}} .
$$

If, in addition, $\left(\tau_{z}\right)_{z \in \mathbb{Z}^{n}}$ (resp. $\left.\left(\tau_{z}\right)_{z \in \mathbb{R}^{n}}\right)$ is ergodic, then $f_{\text {hom }}$ is independent of $\omega$ and

$$
\begin{aligned}
f_{\text {hom }}(\xi) & =\lim _{t \rightarrow+\infty} \frac{1}{t^{n}} \int_{\Omega} m_{F(\omega)}^{1, p}\left(\ell_{\xi}, Q_{t}(0)\right) \mathrm{d} P(\omega) \\
& =\inf _{k \in \mathbb{N}} \frac{1}{k^{n}} \int_{\Omega} m_{F(\omega)}^{1, p}\left(\ell_{\xi}, Q_{k}(0)\right) \mathrm{d} P(\omega) .
\end{aligned}
$$

The proof of Proposition 4.1 relies on the application of the Subadditive Ergodic Theorem 3.11 to the function $(\omega, A) \mapsto m_{F(\omega)}^{1, p}\left(\ell_{\xi}, A\right)$, which is a subadditive process as shown below. 
Proposition 4.2. Let $f$ be a stationary random volume integrand with respect to a group $\left(\tau_{z}\right)_{z \in \mathbb{Z}^{n}}$ (resp. $\left.\left(\tau_{z}\right)_{z \in \mathbb{R}^{n}}\right)$ of P-preserving transformations on $(\Omega, \mathcal{T}, P)$ and let $F(\omega)$ be as in (3.1) with $f(\cdot, \cdot)$ replaced by $f(\omega, \cdot, \cdot)$. Let $\xi \in \mathbb{R}^{m \times n}$ and set

$$
\mu_{\xi}(\omega, A):=m_{F(\omega)}^{1, p}\left(\ell_{\xi}, A\right) \text { for every } \omega \in \Omega, A \in \mathcal{I}_{n}
$$

where $m_{F(\omega)}^{1, p}$ is as in (3.3) and $\mathcal{I}_{n}$ as in (3.9). Then $\mu_{\xi}$ is a subadditive process with respect to $\left(\tau_{z}\right)_{z \in \mathbb{Z}^{n}}$ (resp. $\left.\left(\tau_{z}\right)_{z \in \mathbb{R}^{n}}\right)$ and

$$
0 \leq \mu_{\xi}(\omega, A) \leq c_{2}\left(1+|\xi|^{p}\right) \mathcal{L}^{n}(A) \text { for every } \omega \in \Omega
$$

Proof. See [18] and also [30, Proposition 3.2].

We can now give the proof of Proposition 4.1.

Proof of Proposition 4.1. The existence of $f_{\text {hom }}$ and its independence of $x$ follow from Proposition 4.2 and [18, Theorem 1] (see also [30, Corollary 3.3]). The fact that $f_{\text {hom }}$ is a random volume integrand can be shown arguing as in $[16$, Lemma A.5 and Lemma A.6], and this concludes the proof.

\section{Proof of the Cell-Formula for the Surface Integrand: A Special Case}

This section is devoted to the proof of (3.18) in the the special case $x=0$. Namely, we prove

Theorem 5.1. Let $g$ be a stationary random surface integrand with respect to a group $\left(\tau_{z}\right)_{z \in \mathbb{Z}^{n}}$ (resp. $\left.\left(\tau_{z}\right)_{z \in \mathbb{R}^{n}}\right)$ of P-preserving transformations on $(\Omega, \mathcal{T}, P)$ and let $\widehat{\Omega}$ be the set of all $\omega \in \Omega$ such that the limit

$$
\lim _{\substack{t \rightarrow+\infty \\ t \in \mathbb{Q}}} \frac{m_{G(\omega)}^{\mathrm{pc}}\left(u_{0, \zeta, v}, Q_{t}^{v}(0)\right)}{t^{n-1}}
$$

exists for every $\zeta \in \mathbb{Q}_{0}^{m}$, and $\nu \in \mathbb{Q}^{n} \cap \mathbb{S}^{n-1}$. Then there exist $\widetilde{\Omega} \in \mathcal{T}$, with $\widetilde{\Omega} \subset \widehat{\Omega}$ and $P(\widetilde{\Omega})=1$, and a random surface integrand $g_{\mathrm{hom}}: \Omega \times \mathbb{R}_{0}^{m} \times \mathbb{S}^{n-1} \rightarrow \mathbb{R}$ such that

$$
g_{\text {hom }}(\omega, \zeta, v)=\lim _{t \rightarrow+\infty} \frac{m_{G(\omega)}^{\mathrm{pc}}\left(u_{0, \zeta, \nu}, Q_{t}^{v}(0)\right)}{t^{n-1}},
$$

for every $\omega \in \widetilde{\Omega}, \zeta \in \mathbb{R}_{0}^{m}$, and $v \in \mathbb{S}^{n-1}$.

Remark 5.2. We observe that in general the set $\widehat{\Omega}$ defined in Theorem 5.1 is not $\mathcal{T}$-measurable. 

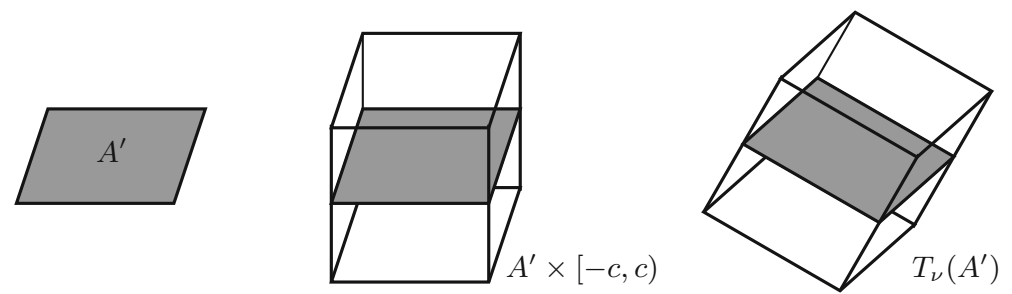

Fig. 1. Construction of the oriented $n$-dimensional interval $T_{v}\left(A^{\prime}\right)$

The proof of Theorem 5.1 will need several preliminary results. A key ingredient will be the application of the Subadditive Ergodic Theorem 3.11 with $k=n-1$. This is a nontrivial task, since it requires to define an $(n-1)$-dimensional subadditive process starting from the $n$-dimensional set function $A \mapsto m_{G(\omega)}^{\mathrm{pc}}\left(u_{0, \zeta, \nu}, A\right)$. To this end, we are now going to illustrate a systematic way to transform $(n-1)$ dimensional intervals (see (3.9)) into $n$-dimensional intervals oriented along a prescribed direction $v \in \mathbb{S}^{n-1}$.

For every $v \in \mathbb{S}^{n-1}$ let $R_{v}$ be the orthogonal $n \times n$ matrix defined in point $(\mathrm{k})$ of Section 2 (see also [16, Example A.1]). Then, the following properties are satisfied:

- $R_{v} e_{n}=v$ for every $v \in \mathbb{S}^{n-1}$

- the restrictions of the function $v \mapsto R_{\nu}$ to the sets $\widehat{\mathbb{S}}_{ \pm}^{n-1}$ are continuous;

- $R_{-v} Q(0)=R_{\nu} Q(0)$ for every $v \in \mathbb{S}^{n-1}$.

Moreover, $R_{v} \in O(n) \cap \mathbb{Q}^{n \times n}$ for every $v \in \mathbb{Q}^{n} \cap \mathbb{S}^{n-1}$. Since $R_{v} e_{n}=v$, we have that $\left\{R_{v} e_{j}\right\}_{j=1, \ldots, n-1}$ is an orthonormal basis of $\Pi_{0}^{v}$. Let now $M_{v}$ be a positive integer such that $M_{\nu} R_{v} \in \mathbb{Z}^{n \times n}$. Note that, in particular, for every $z^{\prime} \in \mathbb{Z}^{n-1}$ we have that $M_{\nu} R_{v}\left(z^{\prime}, 0\right) \in \Pi_{0}^{v} \cap \mathbb{Z}^{n}$, namely $M_{\nu} R_{v}$ maps integer vectors perpendicular to $e_{n}$ into integer vectors perpendicular to $v$.

Let $A^{\prime} \in \mathcal{I}_{n-1}$; we define the (rotated) $n$-dimensional interval $T_{v}\left(A^{\prime}\right)$ as

$$
\begin{aligned}
T_{\nu}\left(A^{\prime}\right) & :=M_{\nu} R_{\nu}\left(A^{\prime} \times[-c, c)\right), \\
c & :=\frac{1}{2} \max _{1 \leq j \leq n-1}\left(b_{j}-a_{j}\right), \quad\left(M_{\nu} R_{\nu} \in \mathbb{Z}^{n \times n}\right),
\end{aligned}
$$

see Fig. 1.

The next proposition is the analogue of Proposition 4.2 for the surface energy, and will be crucial in the proof Theorem 5.1.

Proposition 5.3. Let $g$ be a stationary surface integrand with respect to a group $\left(\tau_{z}\right)_{z \in \mathbb{Z}^{n}}$ (resp. $\left.\left(\tau_{z}\right)_{z \in \mathbb{R}^{n}}\right)$ of P-preserving transformations on $(\Omega, \mathcal{T}, P)$, let $G(\omega)$ be as in (3.2), with $g(\cdot, \cdot, \cdot)$ replaced by $g(\omega, \cdot, \cdot, \cdot)$, let $\zeta \in \mathbb{Q}_{0}^{m}$, and let $v \in$ $\mathbb{Q}^{n} \cap \mathbb{S}^{n-1}$. For every $A^{\prime} \in \mathcal{I}_{n-1}$ and $\omega \in \Omega$ set

$$
\mu_{\zeta, v}\left(\omega, A^{\prime}\right):=\frac{1}{M_{\nu}^{n-1}} m_{G(\omega)}^{\mathrm{pc}}\left(u_{0, \zeta, v}, T_{\nu}\left(A^{\prime}\right)\right),
$$

where $m_{G(\omega)}^{\mathrm{pc}}$ is as in (3.4), while $M_{\nu}$ and $T_{\nu}\left(A^{\prime}\right)$ are as in $(5.2)$. Let $(\Omega, \widehat{\mathcal{T}}, \widehat{P})$ denote the completion of the probability space $(\Omega, \mathcal{T}, P)$. Then there exists a group 
$\left(\tau_{z^{\prime}}^{\nu}\right)_{z^{\prime} \in \mathbb{Z}^{n-1}}$ (resp. $\left.\left(\tau_{z^{\prime}}^{\nu}\right)_{z^{\prime} \in \mathbb{R}^{n-1}}\right)$ of $\widehat{P}$-preserving transformations on $(\Omega, \widehat{\mathcal{T}}, \widehat{P})$ such that $\mu_{\zeta, v}$ is a subadditive process on $(\Omega, \widehat{\mathcal{T}}, \widehat{P})$ with respect to $\left(\tau_{z^{\prime}}^{\nu}\right)_{z^{\prime} \in \mathbb{Z}^{n-1}}$ (resp. $\left(\tau_{z^{\prime}}^{v}\right)_{\left.z^{\prime} \in \mathbb{R}^{n-1}\right) \text {. Moreover }}$

$$
0 \leq \mu_{\zeta, v}(\omega)\left(A^{\prime}\right) \leq c_{4}(1+|\zeta|) \mathcal{L}^{n-1}\left(A^{\prime}\right) \quad \text { for } \widehat{P} \text {-almost everywhere } \omega \in \Omega
$$

Proof. The $\widehat{\mathcal{T}}$-measurability of the function $\omega \mapsto \mu_{\zeta, v}\left(\omega, A^{\prime}\right)$ follows from the $\widehat{\mathcal{T}}$-measurability of $\omega \mapsto m_{G(\omega)}^{\mathrm{pc}}\left(u_{0, \zeta, v}, A\right)$ for every $A \in \mathscr{A}$. This is a delicate issue, which will be postponed to the Appendix.

Let now $\zeta \in \mathbb{Q}_{0}^{m}$, and let $v \in \mathbb{Q}^{n} \cap \mathbb{S}^{n-1}$. By Proposition A.1, for every $A^{\prime} \in \mathcal{I}_{n-1}$ the function $\omega \mapsto \mu_{\zeta, v}\left(\omega, A^{\prime}\right)$ is $\widehat{\mathcal{T}}$-measurable. We are now going to prove that there exists a group $\left(\tau_{z^{\prime}}^{\nu}\right)_{z^{\prime} \in \mathbb{Z}^{n-1}}\left(\operatorname{resp} .\left(\tau_{z^{\prime}}^{\nu}\right)_{z^{\prime} \in \mathbb{R}^{n-1}}\right)$ of $\widehat{P}$-preserving transformations on $(\Omega, \widehat{\mathcal{T}}, \widehat{P})$ such that

$$
\mu_{\zeta, v}\left(\omega, A^{\prime}+z^{\prime}\right)=\mu_{\zeta, v}\left(\tau_{z^{\prime}}^{\nu}(\omega), A^{\prime}\right)
$$

for every $\omega \in \Omega, z^{\prime} \in \mathbb{Z}^{n-1}$ (resp. $z^{\prime} \in \mathbb{R}^{n-1}$ ), and $A^{\prime} \in \mathcal{I}_{n-1}$.

We first consider the case of $g$ stationary with respect to a discrete group $\left(\tau_{z}\right)_{z \in \mathbb{Z}^{n}}$. To this end fix $z^{\prime} \in \mathbb{Z}^{n-1}$ and $A^{\prime} \in \mathcal{I}_{n-1}$. Note that, by (5.2),

$$
\begin{aligned}
T_{\nu}\left(A^{\prime}+z^{\prime}\right) & =M_{\nu} R_{\nu}\left(\left(A^{\prime}+z^{\prime}\right) \times[-c, c)\right) \\
& =M_{\nu} R_{\nu}\left(A^{\prime} \times[-c, c)\right)+M_{\nu} R_{\nu}\left(z^{\prime}, 0\right)=T_{\nu}\left(A^{\prime}\right)+z_{\nu}^{\prime},
\end{aligned}
$$

where $z_{v}^{\prime}:=M_{v} R_{v}\left(z^{\prime}, 0\right) \in \mathbb{Z}^{n}$. Then, by (5.3)

$$
\begin{aligned}
\mu_{\zeta, v}\left(\omega, A^{\prime}+z^{\prime}\right) & =\frac{1}{M_{v}^{n-1}} m_{G(\omega)}^{\mathrm{pc}}\left(u_{0, \zeta, v}, T_{v}\left(A^{\prime}+z^{\prime}\right)\right) \\
& =\frac{1}{M_{v}^{n-1}} m_{G(\omega)}^{\mathrm{pc}}\left(u_{0, \zeta, v}, T_{v}\left(A^{\prime}\right)+z_{v}^{\prime}\right) .
\end{aligned}
$$

Given $u \in L^{0}\left(\mathbb{R}^{n}, \mathbb{R}^{m}\right)$, let $v \in L^{0}\left(\mathbb{R}^{n}, \mathbb{R}^{m}\right)$ be defined by $v(x):=u\left(x+z_{v}^{\prime}\right)$ for every $x \in \mathbb{R}^{n}$. By a change of variables we have

$$
\begin{gathered}
\int_{S_{u} \cap\left(T_{v}\left(A^{\prime}\right)+z_{v}^{\prime}\right)} g\left(\omega, x,[u], v_{u}\right) \mathrm{d} \mathcal{H}^{n-1}(x) \\
=\int_{S_{v} \cap T_{v}\left(A^{\prime}\right)} g\left(\omega, y+z_{v}^{\prime},[v], v_{v}\right) \mathrm{d} \mathcal{H}^{n-1}(y) .
\end{gathered}
$$

Since $z_{v}^{\prime} \in \mathbb{Z}^{n}$, by the stationarity of $g$ we have also $g\left(\omega, y+z_{v}^{\prime},[v], v_{v}\right)=$ $g\left(\tau_{z_{v}^{\prime}}(\omega), y,[v], v_{v}\right)$. From these equalities we obtain

$$
G(\omega)\left(u, \operatorname{int} T_{v}\left(A^{\prime}\right)+z_{v}^{\prime}\right)=G\left(\tau_{z_{v}^{\prime}}(\omega)\right)\left(v, \operatorname{int} T_{v}\left(A^{\prime}\right)\right) .
$$

Since $z_{v}^{\prime}$ is perpendicular to $v$, we have $u_{0, \zeta, v}(x)=u_{0, \zeta, v}\left(x+z_{v}^{\prime}\right)$ for every $x \in \mathbb{R}^{n}$. Therefore, from (3.4), (5.5), and (5.6) we obtain that $\mu_{\zeta, v}\left(\omega, A^{\prime}+z^{\prime}\right)=$ $\mu_{\zeta, v}\left(\tau_{z_{v}^{\prime}}(\omega), A^{\prime}\right)$. Thus, $\mu_{\zeta, v}$ is covariant with respect to the group $\left(\tau_{z^{\prime}}^{v}\right)_{z^{\prime} \in \mathbb{Z}^{n-1}}$ of $\widehat{P}$-preserving transformations on $(\Omega, \widehat{\mathcal{T}}, \widehat{P})$ defined by 

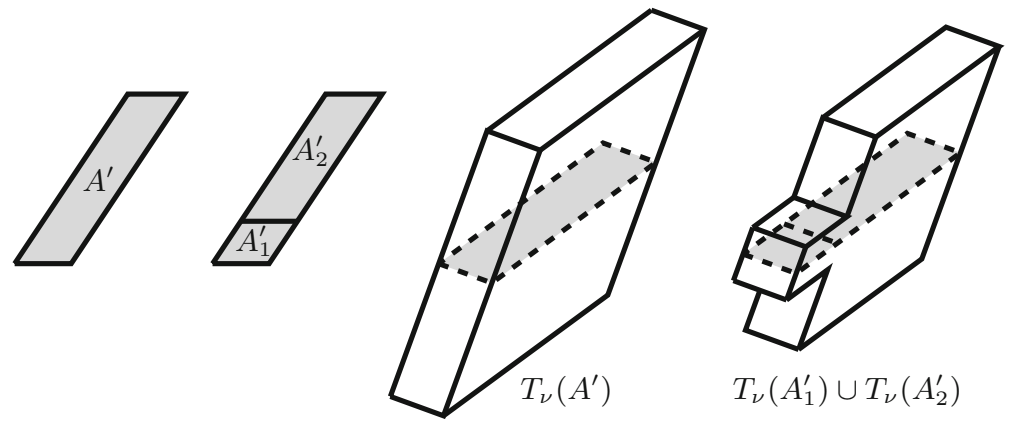

Fig. 2. An example with $N=2$ in which $T_{\nu}\left(A^{\prime}\right) \neq T_{\nu}\left(A_{1}^{\prime}\right) \cup T_{\nu}\left(A_{2}^{\prime}\right)$

$$
\left(\tau_{z^{\prime}}^{v}\right)_{z^{\prime} \in \mathbb{Z}^{n-1}}:=\left(\tau_{z_{v}^{\prime}}\right)_{z^{\prime} \in \mathbb{Z}^{n-1}}
$$

Note that if $g$ is stationary with respect to a continuous group $\left(\tau_{z}\right)_{z \in \mathbb{R}^{n}}$, then the same construction as above provides, for fixed $z^{\prime} \in \mathbb{R}^{n-1}$, a vector $z_{v}^{\prime} \in \mathbb{R}^{n}$ such that $\mu_{\zeta, v}$ is covariant with respect to the group of $P$-preserving transformations defined as in (5.7), with $\mathbb{Z}^{n-1}$ replaced by $\mathbb{R}^{n-1}$. Moreover, in this case one can simply define $z_{v}^{\prime}:=R_{v}\left(z^{\prime}, 0\right)$, namely the multiplication by the positive integer $M_{v}$ is not needed.

We now show that $\mu_{\zeta, v}$ is subadditive. To this end let $A^{\prime} \in \mathcal{I}_{n-1}$ and let $\left(A_{i}^{\prime}\right)_{1 \leq i \leq N} \subset \mathcal{I}_{n-1}$ be a finite family of pairwise disjoint sets such that $A^{\prime}=\bigcup_{i} A_{i}^{\prime}$. For fixed $\eta>0$ and $i=1, \ldots, N$, let $u_{i} \in S B V_{\mathrm{pc}}\left(\operatorname{int} T_{\nu}\left(A_{i}^{\prime}\right)\right)$ be such that $u_{i}=u_{0, \zeta, v}$ in a neighbourhood of $\partial T_{\nu}\left(A_{i}^{\prime}\right)$ and

$$
G(\omega)\left(u_{i}, \operatorname{int} T_{\nu}\left(A_{i}\right)\right) \leq m_{G(\omega)}^{\mathrm{pc}}\left(u_{0, \zeta, v}, T_{\nu}\left(A_{i}^{\prime}\right)\right)+\eta .
$$

Note that $T_{v}\left(A^{\prime}\right)$ can differ from $\bigcup_{i} T_{v}\left(A_{i}^{\prime}\right)$ but, by construction, we always have $\bigcup_{i} T_{\nu}\left(A_{i}^{\prime}\right) \subset T_{\nu}\left(A^{\prime}\right)$ (see Fig. 2).

Now we define

$$
u(y):= \begin{cases}u_{i}(y) & \text { if } y \in T_{v}\left(A_{i}^{\prime}\right), i=1, \ldots, N, \\ u_{0, \zeta, v}(y) & \text { if } y \in T_{\nu}\left(A^{\prime}\right) \backslash \bigcup_{i} T_{\nu}\left(A_{i}^{\prime}\right) ;\end{cases}
$$

then $u \in S B V_{\mathrm{pc}}\left(\operatorname{int} T_{v}\left(A^{\prime}\right)\right)$ and $u=u_{0, \zeta, v}$ in a neighbourhood of $\partial T_{v}\left(A^{\prime}\right)$. Moreover, by the additivity and the locality of $G(\omega)$ we have

$$
\begin{aligned}
G(\omega)\left(u, \operatorname{int} T_{\nu}\left(A^{\prime}\right)\right)= & \sum_{i=1}^{N} G(\omega)\left(u_{i}, \operatorname{int} T_{\nu}\left(A_{i}^{\prime}\right)\right) \\
& +G(\omega)\left(u_{0, \zeta, v}, \operatorname{int}\left(T_{\nu}\left(A^{\prime}\right) \backslash \bigcup_{i} T_{\nu}\left(A_{i}^{\prime}\right)\right)\right),
\end{aligned}
$$

where we have also used the fact that $S_{u} \cap \partial T_{\nu}\left(A_{i}^{\prime}\right)=\emptyset$ for every $i=1, \ldots, N$. Note that the last term in (5.9) is equal to zero because the jump set of $u_{0, \zeta, v}$ is the hyperplane $\Pi_{0}^{v}$, which does not intersect $T_{v}\left(A^{\prime}\right) \backslash \bigcup_{i} T_{v}\left(A_{i}^{\prime}\right)$; therefore

$$
G(\omega)\left(u, \operatorname{int} T_{v}\left(A^{\prime}\right)\right)=\sum_{i=1}^{N} G(\omega)\left(u_{i}, \operatorname{int} T_{v}\left(A_{i}^{\prime}\right)\right) .
$$


As a consequence, by (5.8),

$$
m_{G(\omega)}^{\mathrm{pc}}\left(u_{0, \zeta, \nu}, T_{\nu}\left(A^{\prime}\right)\right) \leq \sum_{i=1}^{N} m_{G(\omega)}^{\mathrm{pc}}\left(u_{0, \zeta, \nu}, T_{\nu}\left(A_{i}^{\prime}\right)\right)+N \eta,
$$

thus the subadditivity of $\mu_{\zeta, v}$ follows from (5.3) and from the arbitrariness of $\eta$.

Finally, in view of $(g 6)$ for every $A^{\prime} \in \mathcal{I}_{n-1}$ we have

$$
\begin{aligned}
\mu_{\zeta, v}\left(\omega, A^{\prime}\right) & \leq \frac{1}{M_{v}^{n-1}} G(\omega)\left(u_{0, \zeta, v}, \operatorname{int} T_{\nu}\left(A^{\prime}\right)\right) \\
& \leq \frac{c_{5}(1+|\zeta|)}{M_{v}^{n-1}} \mathcal{H}^{n-1}\left(\Pi_{0}^{v} \cap T_{v}\left(A^{\prime}\right)\right) \\
& =c_{5}(1+|\zeta|) \mathcal{L}^{n-1}\left(A^{\prime}\right),
\end{aligned}
$$

and thus (5.4).

To conclude the proof of Proposition 5.1 we need two preliminary lemmas.

Lemma 5.4. Let $g \in \mathcal{G}$, let $G$ be as in (3.2), and let $m_{G}^{\mathrm{pc}}$ be as in (3.4). Let $\underline{g}$, $\bar{g}: \mathbb{R}_{0}^{m} \times \mathbb{S}^{n-1} \rightarrow[-\infty,+\infty]$ be the functions defined by

$$
\begin{aligned}
& \underline{g}(\zeta, v):=\liminf _{t \rightarrow+\infty} \frac{m_{G}^{\mathrm{pc}}\left(u_{0, \zeta, v}, Q_{t}^{v}(0)\right)}{t^{n-1}} \text { and } \\
& \bar{g}(\zeta, v):=\limsup _{t \rightarrow+\infty} \frac{m_{G}^{\mathrm{pc}}\left(u_{0, \zeta, v}, Q_{t}^{v}(0)\right)}{t^{n-1}} .
\end{aligned}
$$

Then $\underline{g}, \bar{g} \in \mathcal{G}$.

Proof. It is enough to adapt the proof of [16, Lemma A.7].

We will also need the following result.

Lemma 5.5. Let $g \in \mathcal{G}$, let $G$ be as in (3.2), and let $m_{G}^{\mathrm{pc}}$ be as in (3.4). Let $g, \underset{\sim}{\tilde{g}}: \mathbb{R}^{n} \times$ $\mathbb{R}_{0}^{m} \times \mathbb{S}^{n-1} \rightarrow[-\infty,+\infty]$ be the functions defined by

$$
g(x, \zeta, v):=\liminf _{t \rightarrow+\infty} \frac{m_{G}^{\mathrm{pc}}\left(u_{t x, \zeta, v}, Q_{t}^{v}(t x)\right)}{t^{n-1}}
$$

and

$$
\widetilde{g}(x, \zeta, v):=\limsup _{t \rightarrow+\infty} \frac{m_{G}^{\mathrm{pc}}\left(u_{t x, \zeta, v}, Q_{t}^{v}(t x)\right)}{t^{n-1}}
$$

Then $g$ and $\tilde{g}$ satisfy ( $g 2$ ). Moreover for every $x \in \mathbb{R}^{n}$ and $\zeta \in \mathbb{R}_{0}^{m}$ the restriction of the functions $v \mapsto g(x, \zeta, v)$ and $v \mapsto \widetilde{g}(x, \zeta, v)$ to the sets $\widehat{\mathbb{S}}_{+}^{n-1}$ and $\widehat{\mathbb{S}}_{-}^{n-1}$ are continuous. 
Proof. The proof of $(g 2)$ can be obtained by adapting the proof of [16, Lemma A.7].

To prove the continuity of $v \mapsto g(x, \zeta, v)$ on $\widehat{\mathbb{S}}_{+}^{n-1}$, we fix $x \in \mathbb{R}^{n}, \zeta \in \mathbb{R}_{0}^{m}$, $v \in \widehat{\mathbb{S}}_{+}^{n-1}$, and a sequence $\left(v_{j}\right) \subset \widehat{\mathbb{S}}_{+}^{n-1}$ such that $v_{j} \rightarrow v$ as $j \rightarrow+\infty$. Since the function $v \mapsto R_{v}$ is continuous on $\widehat{\mathbb{S}}_{+}^{n-1}$, for every $\delta \in\left(0, \frac{1}{2}\right)$ there exists an integer $j_{\delta}$ such that

$$
Q_{(1-\delta) t}^{v_{j}}(t x) \subset \subset Q_{t}^{v}(t x) \subset \subset Q_{(1+\delta) t}^{v_{j}}(t x),
$$

for every $j \geq j_{\delta}$ and every $t>0$. Fix $j \geq j_{\delta}, t>0$, and $\eta>0$. Let $u \in$ $S B V_{\mathrm{pc}}\left(Q_{t}^{v}(t x), \mathbb{R}^{m}\right)$ be such that $u=u_{t x, \zeta, v}$ in a neighbourhood of $\partial Q_{t}^{v}(t x)$, and

$$
G\left(u, Q_{t}^{v}(t x)\right) \leq m_{G}^{\mathrm{pc}}\left(u_{t x, \zeta, v}, Q_{t}^{v}(t x)\right)+\eta .
$$

We set

$$
v(y):= \begin{cases}u(y) & \text { if } y \in Q_{t}^{v}(t x), \\ u_{t x, \zeta, v_{j}}(y) & \text { if } y \in Q_{(1+\delta) t}^{v_{j}}(t x) \backslash Q_{t}^{v}(t x) .\end{cases}
$$

Then $v \in S B V_{\mathrm{pc}}\left(Q_{(1+\delta) t}^{v_{j}}(t x), \mathbb{R}^{m}\right), v=u_{t x, \zeta, v}$ in a neighbourhood of $\partial Q_{(1+\delta) t}^{v_{j}}$ $(t x)$, and $S_{v} \subset S_{u} \cup \Sigma$, where

$$
\Sigma:=\left\{y \in \partial Q_{t}^{v}(t x):((y-t x) \cdot v)\left((y-t x) \cdot v_{j}\right)<0\right\} \cup \Pi_{t x}^{v_{j}} \cap\left(Q_{(1+\delta) t}^{v_{j}}(t x) \backslash Q_{t}^{v}(t x)\right) .
$$

By (5.12) there exists $\varsigma(\delta)>0$, independent of $j$ and $t$, with $\varsigma(\delta) \rightarrow 0$ as $\delta \rightarrow 0+$, such that $\mathcal{H}^{n-1}(\Sigma) \leq \varsigma(\delta) t^{n-1}$. Thanks to $(g 6)$ we then have

$$
\begin{aligned}
m_{G}^{\mathrm{pc}}\left(u_{t x, \zeta, v_{j}}, Q_{(1+\delta) t}^{v_{j}}(t x)\right) \leq & G\left(v, Q_{(1+\delta) t}^{v_{j}}(t x)\right) \leq G\left(u, Q_{t}^{v}(t x)\right) \\
& +\varsigma(\delta) c_{5}(1+|\zeta|) t^{n-1} \\
\leq & m_{G}^{\mathrm{pc}}\left(u_{t x, \zeta, v}, Q_{t}^{v}(t x)\right)+\eta+\varsigma(\delta) c_{5}(1+|\zeta|) t^{n-1}
\end{aligned}
$$

By dividing the terms of the above estimate by $t^{n-1}$ and passing to the liminf as $t \rightarrow+\infty$, from (5.10) we obtain that

$$
\underset{\sim}{g}\left(x, \zeta, v_{j}\right)(1+\delta)^{n-1} \leq \underset{\sim}{g}(x, \zeta, v)+\varsigma(\delta) c_{5}(1+|\zeta|) .
$$

Letting $j \rightarrow+\infty$ and then $\delta \rightarrow 0+$ we deduce that

$$
\limsup _{j \rightarrow+\infty} g\left(x, \zeta, v_{j}\right) \leq g(x, \zeta, v) \text {. }
$$

An analogous argument, now using the cube $Q_{(1-\delta) t}^{v_{j}}(t x)$, yields

$$
g(x, \zeta, v) \leq \liminf _{j \rightarrow+\infty} g\left(x, \zeta, v_{j}\right)
$$

and hence the continuity of $\underset{\sim}{g}(x, \zeta, \cdot)$ in $\widehat{\mathbb{S}}_{+}^{n-1}$. The proof of the continuity in $\widehat{\mathbb{S}}_{-}^{n-1}$, as well as that of the continuity of $\tilde{g}$ are similar.

We are now ready to prove Theorem 5.1. 
Proof of Theorem 5.1. Let $(\Omega, \widehat{\mathcal{T}}, \widehat{P})$ be the completion of the probability space $(\Omega, \mathcal{T}, P)$. By Proposition A.1 for $\zeta \in \mathbb{Q}_{0}^{m}$ and $\nu \in \mathbb{Q}^{n} \cap \mathbb{S}^{n-1}$ fixed the function $\omega \mapsto m_{G(\omega)}^{\mathrm{pc}}\left(u_{0, \zeta, v}, Q_{t}^{v}(0)\right)$ is $\widehat{\mathcal{T}}$-measurable for every $t>0$, hence $\widehat{\Omega} \in \widehat{\mathcal{T}}$. We apply the Subadditive Ergodic Theorem 3.11 to the subadditive process $\mu_{\zeta, v}$ defined on $(\Omega, \widehat{\mathcal{T}}, \widehat{P})$ by (5.3). Choosing $Q^{\prime}:=\left[-\frac{1}{2}, \frac{1}{2}\right)^{n-1}$, we obtain the existence of a set $\widehat{\Omega}_{\zeta, v} \in \widehat{\mathcal{T}}$, with $\widehat{P}\left(\widehat{\Omega}_{\zeta, v}\right)=1$, and of a $\widehat{\mathcal{T}}$-measurable function $g_{\zeta, v}: \Omega \rightarrow \mathbb{R}$ such that

$$
\lim _{t \rightarrow+\infty} \frac{\mu_{\zeta, v}(\omega)\left(t Q^{\prime}\right)}{t^{n-1}}=g_{\zeta, v}(\omega)
$$

for every $\omega \in \widehat{\Omega}_{\zeta, \nu}$. Then, by the properties of the completion there exist a set $\Omega_{\zeta, v} \in \mathcal{T}$, with $P\left(\Omega_{\zeta, v}\right)=1$, and a $\mathcal{T}$-measurable function, which we still denote by $g_{\zeta, v}$, such that (5.13) holds for every $\omega \in \Omega_{\zeta, v}$. Using the definition of $\mu_{\zeta, v}$ we then have

$$
g_{\zeta, \nu}(\omega)=\lim _{t \rightarrow+\infty} \frac{m_{G(\omega)}^{\mathrm{pc}}\left(u_{0, \zeta, v}, t T_{\nu}\left(Q^{\prime}\right)\right)}{M_{\nu}^{n-1} t^{n-1}}=\lim _{t \rightarrow+\infty} \frac{m_{G(\omega)}^{\mathrm{pc}}\left(u_{0, \zeta, v}, t M_{\nu} Q^{\nu}(0)\right)}{\left(t M_{\nu}\right)^{n-1}}
$$

for every $\omega \in \Omega_{\zeta, v}$. Let $\widetilde{\Omega}$ be the intersection of the sets $\Omega_{\zeta, v}$ for $\zeta \in \mathbb{Q}_{0}^{m}$ and $v \in \mathbb{Q}^{n} \cap \mathbb{S}^{n-1}$. Clearly $\widetilde{\Omega} \in \mathcal{T}$ and $P(\widetilde{\Omega})=1$.

We now consider the auxiliary functions $\underline{g}, \bar{g}: \widetilde{\Omega} \times \mathbb{R}_{0}^{m} \times \mathbb{S}^{n-1} \rightarrow[0,+\infty]$ defined as

$$
\begin{aligned}
& \underline{g}(\omega, \zeta, v):=\liminf _{t \rightarrow+\infty} \frac{m_{G(\omega)}^{\mathrm{pc}}\left(u_{0, \zeta, v}, Q_{t}^{v}(0)\right)}{t^{n-1}}, \\
& \bar{g}(\omega, \zeta, v):=\limsup _{t \rightarrow+\infty} \frac{m_{G(\omega)}^{\mathrm{pc}}\left(u_{0, \zeta, v}, Q_{t}^{v}(0)\right)}{t^{n-1}},
\end{aligned}
$$

and note that $\underline{g}(\omega, \zeta, v)=\bar{g}(\omega, \zeta, v)=g_{\zeta, v}(\omega)$ for every $\omega \in \widetilde{\Omega}, \zeta \in \mathbb{Q}_{0}^{m}$, and $v \in \mathbb{Q}^{n} \cap \mathbb{S}^{n-\overline{1}}$, hence $\widetilde{\Omega} \subset \widehat{\Omega}$.

By Lemma 5.4 for every $\omega \in \widetilde{\Omega}$ and every $v \in \mathbb{S}^{n-1}$ the functions $\zeta \mapsto$ $\underline{g}(\omega, \zeta, v)$ and $\zeta \mapsto \bar{g}(\omega, \zeta, v)$ are continuous on $\mathbb{R}_{0}^{m}$, and their modulus of continuity does not depend on $\omega$ and $\nu$. This implies that

$$
\underline{g}(\omega, \zeta, v)=\bar{g}(\omega, \zeta, v) \text { for every } \omega \in \widetilde{\Omega}, \zeta \in \mathbb{R}_{0}^{m}, \text { and } v \in \mathbb{Q}^{n} \cap \mathbb{S}^{n-1},
$$

and that the function $\omega \mapsto \bar{g}(\omega, \zeta, v)$ is $\mathcal{T}$-measurable on $\widetilde{\Omega}$ for every $\zeta \in \mathbb{R}_{0}^{m}$ and $v \in \mathbb{Q}^{n} \cap \mathbb{S}^{n-1}$.

Let $\mathbb{S}_{ \pm}^{n-1}$ and $\widehat{\mathbb{S}}_{ \pm}^{n-1}$ be the sets defined in (b), Section 2 . It is known that $\mathbb{Q}^{n} \cap \mathbb{S}^{n-1}$ is dense in $\mathbb{S}^{n-1}$ (see, for example, [16, Remark A.2]). Since $\mathbb{S}_{ \pm}^{n-1}$ is open in the relative topology of $\mathbb{S}^{n-1}$ and is dense in $\widehat{\mathbb{S}}_{ \pm}^{n-1}$, we conclude that $\mathbb{Q}^{n} \cap \mathbb{S}_{ \pm}^{n-1}$ is dense in $\widehat{\mathbb{S}}_{ \pm}^{n-1}$.

Since, for fixed $\omega \in \widetilde{\Omega}$, the function $g$ in (5.14) coincides with $g$ in (5.10) (for $G=G(\omega)$ ) evaluated at $x=0$, while $\bar{g}$ in (5.15) coincides with $\widetilde{g}$ in (5.11) (for $G=G(\omega))$ evaluated at $x=0$, by Lemma 5.5, for every $\omega \in \widetilde{\Omega}$ and $\zeta \in \mathbb{R}_{0}^{m}$ the 
restrictions of the functions $v \mapsto g(\omega, \zeta, v)$ and $v \mapsto \bar{g}(\omega, \zeta, v)$ to the sets $\widehat{\mathbb{S}}_{+}^{n-1}$ and $\widehat{\mathbb{S}}_{-}^{n-1}$ are continuous. Therefore (5.16) and the density of $\mathbb{Q}^{n} \cap \mathbb{S}_{ \pm}^{n-1}$ in $\widehat{\mathbb{S}}_{ \pm}^{n-1}$ imply that

$$
\underline{g}(\omega, \zeta, v)=\bar{g}(\omega, \zeta, v) \text { for every } \omega \in \widetilde{\Omega}, \zeta \in \mathbb{R}_{0}^{m}, \text { and } v \in \mathbb{S}^{n-1},
$$

and that the function $\omega \mapsto \bar{g}(\omega, \zeta, v)$ is $\mathcal{T}$-measurable on $\widetilde{\Omega}$ for every $\zeta \in \mathbb{R}_{0}^{m}$ and $v \in \mathbb{S}^{n-1}$.

For every $\omega \in \Omega, \zeta \in \mathbb{R}_{0}^{m}$, and $v \in \mathbb{S}^{n-1}$ we define

$$
g_{\text {hom }}(\omega, \zeta, v)= \begin{cases}\bar{g}(\omega, \zeta, v) & \text { if } \omega \in \widetilde{\Omega}, \\ c_{4} & \text { if } \omega \in \Omega \backslash \widetilde{\Omega}\end{cases}
$$

By (5.17) we may deduce (5.1) for every $\omega \in \widetilde{\Omega}, \zeta \in \mathbb{R}_{0}^{m}$, and $v \in \mathbb{S}^{n-1}$. Moreover, we have proved that

$\omega \mapsto \bar{g}(\omega, \zeta, v)$ is $\mathcal{T}$-measurable in $\widetilde{\Omega}$ for every $\zeta \in \mathbb{R}_{0}^{m}$ and $v \in \mathbb{S}^{n-1}$, $(\zeta, v) \mapsto \bar{g}(\omega, \zeta, v)$ is continuous in $\mathbb{R}_{0}^{m} \times \widehat{\mathbb{S}}_{ \pm}^{n-1} \quad$ for every $\omega \in \widetilde{\Omega}$.

Therefore the $\mathcal{T}$-measurability of the function $\omega \mapsto \bar{g}(\omega, \zeta, \nu)$ in $\widetilde{\Omega}$ for every $\zeta \in$ $\mathbb{R}_{0}^{m}$ and $v \in \mathbb{S}^{n-1}$ implies that the restriction of $\bar{g}$ to $\widetilde{\Omega} \times \mathbb{R}_{0}^{m} \times \widehat{\mathbb{S}}_{ \pm}^{n-1}$ is measurable with respect to the $\sigma$-algebra induced in $\widetilde{\Omega} \times \mathbb{R}_{0}^{m} \times \widehat{\mathbb{S}}_{ \pm}^{n-1}$ by $\mathcal{T} \otimes \mathscr{B}^{m} \otimes \mathscr{B}_{S}^{n}$. This implies the $\left(\mathcal{T} \otimes \mathscr{B}^{m} \otimes \mathscr{B}_{S}^{n}\right)$-measurability of $g_{\text {hom }}$ on $\Omega \times \mathbb{R}_{0}^{m} \times \mathbb{S}^{n-1}$, thus showing that $g_{\text {hom }}$ satisfies property (c) of Definition 3.5.

Note now that for every $\omega \in \Omega$ the function $(x, \zeta, \nu) \mapsto g_{\text {hom }}(\omega, \zeta, \nu)$ defined in (5.18) belongs to the class $\mathcal{G}$. Indeed, for $\omega \in \widetilde{\Omega}$ this follows from Lemma 5.4 while for $\omega \in \Omega \backslash \widetilde{\Omega}$ this follows from the definition of $g_{\text {hom }}$. Thus, $g_{\text {hom }}$ satisfies property (d) of Definition 3.5, and this concludes the proof.

\section{Proof of the Formula for the Surface Integrand: The General Case}

In this section we extend Theorem 5.1 to the case of arbitrary $x \in \mathbb{R}^{n}$, thus concluding the proof of (3.18). More precisely, we prove the following result.

Theorem 6.1. Let $g$ be a stationary random surface integrand with respect to a group $\left(\tau_{z}\right)_{z \in \mathbb{Z}^{n}}$ (resp. $\left.\left(\tau_{z}\right)_{z \in \mathbb{R}^{n}}\right)$ of P-preserving transformations on $(\Omega, \mathcal{T}, P)$. Then there exist $\Omega^{\prime} \in \mathcal{T}$, with $P\left(\Omega^{\prime}\right)=1$, and a random surface integrand $g_{\text {hom }}: \Omega \times \mathbb{R}_{0}^{m} \times \mathbb{S}^{n-1} \rightarrow \mathbb{R}$, independent of $x$, such that

$$
g_{\text {hom }}(\omega, \zeta, v)=\lim _{t \rightarrow+\infty} \frac{m_{G(\omega)}^{\mathrm{pc}}\left(u_{t x, \zeta, v}, Q_{r(t)}^{v}(t x)\right)}{r(t)^{n-1}},
$$


for every $\omega \in \Omega^{\prime}, x \in \mathbb{R}^{n}, \zeta \in \mathbb{R}_{0}^{m}, v \in \mathbb{S}^{n-1}$, and for every function $r:(0,+\infty) \rightarrow$ $(0,+\infty)$ with $r(t) \geq t$ for $t>0$. Moreover, if $\left(\tau_{z}\right)_{z \in \mathbb{Z}^{n}}$ (resp. $\left.\left(\tau_{z}\right)_{z \in \mathbb{R}^{n}}\right)$ is ergodic, then $g_{\text {hom }}$ does not depend on $\omega$ and

$$
g_{\text {hom }}(\zeta, v)=\lim _{t \rightarrow+\infty} \frac{1}{r(t)^{n-1}} \int_{\Omega} m_{G(\omega)}^{\mathrm{pc}}\left(u_{0, \zeta, v}, Q_{r(t)}^{v}(0)\right) \mathrm{d} P(\omega) .
$$

The first step in the proof of the above statement is the following invariance result. In the ergodic case this implies that the function $g_{\text {hom }}$ does not depend on $\omega$ (see Corollary 6.3).

Theorem 6.2. Let $g$ be a stationary random surface integrand with respect to a group $\left(\tau_{z}\right)_{z \in \mathbb{Z}^{n}}$ (resp. $\left.\left(\tau_{z}\right)_{z \in \mathbb{R}^{n}}\right)$ of P-preserving transformations on $(\Omega, \mathcal{T}, P)$, and let $\widehat{\Omega}, \widetilde{\Omega}$, and $g_{\text {hom }}$ be as in Theorem 5.1. Then for every $z \in \mathbb{Z}^{n}$ (resp. $z \in \mathbb{R}^{n}$ ) we have $\tau_{z}(\widehat{\Omega})=\widehat{\Omega}, P\left(\widetilde{\Omega} \cap \tau_{-z}(\widetilde{\Omega})\right)=1$, and

$$
g_{\text {hom }}\left(\tau_{z}(\omega), \zeta, v\right)=g_{\text {hom }}(\omega, \zeta, v)
$$

for every $\omega \in \widetilde{\Omega} \cap \tau_{-z}(\widetilde{\Omega}), \zeta \in \mathbb{R}_{0}^{m}$, and $\nu \in \mathbb{S}^{n-1}$.

Proof. We prove the theorem when the set of indices is $\mathbb{R}^{n}$, the other case being easier. To obtain the equality $\tau_{z}(\widehat{\Omega})=\widehat{\Omega}$ it is enough to prove the inclusion $\tau_{z}(\widehat{\Omega}) \subset$ $\widehat{\Omega}$. The opposite one can be obtained by using the group properties of $\left(\tau_{z}\right)_{z \in \mathbb{R}^{n}}$.

Let $z \in \mathbb{R}^{n}, \omega \in \Omega, \zeta \in \mathbb{R}_{0}^{m}$, and $v \in \mathbb{S}^{n-1}$ be fixed. Since $g$ is stationary, using (3.8) and a change of variables for every $t>0$ we obtain

$$
m_{G\left(\tau_{z}(\omega)\right)}^{\mathrm{pc}}\left(u_{0, \zeta, \nu}, Q_{t}^{v}(0)\right)=m_{G(\omega)}^{\mathrm{pc}}\left(u_{z, \zeta, \nu}, Q_{t}^{v}(z)\right) .
$$

For every $t>3|z|$, let $u_{t} \in S B V_{\mathrm{pc}}\left(Q_{t}^{v}(0), \mathbb{R}^{m}\right)$ be such that $u_{t}=u_{0, \zeta, v}$ in a neighbourhood of $\partial Q_{t}^{v}(0)$, and

$$
G(\omega)\left(u_{t}, Q_{t}^{v}(0)\right) \leq m_{G(\omega)}^{\mathrm{pc}}\left(u_{0, \zeta, v}, Q_{t}^{v}(0)\right)+1 .
$$

We now modify $u_{t}$ to obtain a competitor for a minimisation problem related to the right-hand side of (6.4). Noting that $Q_{t}^{v}(0) \subset \subset Q_{t+3|z|}^{v}(z)$ we define

$$
v_{t}(y):= \begin{cases}u_{t}(y) & \text { if } y \in Q_{t}^{v}(0), \\ u_{z, \zeta, v}(y) & \text { if } y \in Q_{t+3|z|}^{v}(z) \backslash Q_{t}^{v}(0) .\end{cases}
$$

Clearly $v_{t} \in S B V_{\mathrm{pc}}\left(Q_{t+3|z|}^{v}(z), \mathbb{R}^{m}\right)$ and $v_{t}=u_{z, \zeta, v}$ in a neighbourhood of $\partial Q_{t+3|z|}^{v}(z)$. It is easy to see that $S_{v_{t}}=S_{u_{t}} \cup \Sigma_{1} \cup \Sigma_{2}$, where

$$
\begin{aligned}
& \Sigma_{1}:=\left\{y \in \partial Q_{t}^{v}(0):(y \cdot v)((y-z) \cdot v)<0\right\} \quad \text { and } \\
& \Sigma_{2}:=\Pi_{z}^{v} \cap\left(Q_{t+3|z|}^{v}(z) \backslash Q_{t}^{v}(0)\right) .
\end{aligned}
$$

Moreover $\left|\left[v_{t}\right]\right|=|\zeta| \mathcal{H}^{n-1}$-almost everywhere on $\Sigma_{1} \cup \Sigma_{2}$. Since $3|z|<t$, we have $\mathcal{H}^{n-1}\left(\Sigma_{1}\right)=2(n-1)|z \cdot v| t^{n-2}$ and $\mathcal{H}^{n-1}\left(\Sigma_{2}\right)=(t+3|z|)^{n-1}-t^{n-1} \leq$ $3(n-1)|z|(t+3|z|)^{n-2}<2^{n}(n-1)|z| t^{n-2}$. Therefore $(g 6)$ gives

$$
G(\omega)\left(v_{t}, Q_{t+3|z|}^{v}(z)\right) \leq G(\omega)\left(u_{t}, Q_{t}^{v}(0)\right)+M_{\zeta, z} t^{n-2},
$$


where $M_{\zeta, z}:=c_{5}(n-1)\left(2+2^{n}\right)|z|(1+|\zeta|)$. This inequality, combined with (6.5) and with the definition of $m_{G(\omega)}^{\mathrm{pc}}$, gives

$$
m_{G(\omega)}^{\mathrm{pc}}\left(u_{z, \zeta, v}, Q_{t+3|z|}^{v}(z)\right) \leq m_{G(\omega)}^{\mathrm{pc}}\left(u_{0, \zeta, \nu}, Q_{t}^{v}(0)\right)+1+M_{\zeta, z} t^{n-2} .
$$

Using (6.4), with $t$ replaced by $t+3|z|$, from the inequality above we obtain

$$
m_{G\left(\tau_{z}(\omega)\right)}^{\mathrm{pc}}\left(u_{0, \zeta, \nu}, Q_{t+3|z|}^{v}(0)\right) \leq m_{G(\omega)}^{\mathrm{pc}}\left(u_{0, \zeta, \nu}, Q_{t}^{\nu}(0)\right)+1+M_{\zeta, z} t^{n-2} .
$$

The same inequality, with $\omega$ replaced by $\tau_{z}(\omega)$ and $z$ replaced by $-z$, gives

$$
m_{G(\omega)}^{\mathrm{pc}}\left(u_{0, \zeta, \nu}, Q_{t+3|z|}^{v}(0)\right) \leq m_{G\left(\tau_{z}(\omega)\right)}^{\mathrm{pc}}\left(u_{0, \zeta, \nu}, Q_{t}^{v}(0)\right)+1+M_{\zeta, z} t^{n-2} .
$$

Since $t^{n-1} /(t+3|z|)^{n-1} \rightarrow 1$ as $t \rightarrow+\infty$, dividing by $t^{n-1}$ we obtain

$$
\begin{gathered}
\limsup _{\substack{t \rightarrow+\infty \\
t \in \mathbb{Q}}} \frac{m_{G\left(\tau_{z}(\omega)\right)}^{\mathrm{pc}}\left(u_{0, \zeta, v}, Q_{t}^{v}(0)\right)}{t^{n-1}} \leq \limsup _{\substack{t \rightarrow+\infty \\
t \in \mathbb{Q}}} \frac{m_{G(\omega)}^{\mathrm{pc}}\left(u_{0, \zeta, v}, Q_{t}^{v}(0)\right)}{t^{n-1}}, \\
\liminf _{\substack{t \rightarrow+\infty \\
t \in \mathbb{Q}}} \frac{m_{G(\omega)}^{\mathrm{pc}}\left(u_{0, \zeta, v}, Q_{t}^{v}(0)\right)}{t^{n-1}} \leq \liminf _{\substack{t \rightarrow+\infty \\
t \in \mathbb{Q}}} \frac{m_{G\left(\tau_{z}(\omega)\right)}^{\mathrm{pc}}\left(u_{0, \zeta, v}, Q_{t}^{v}(0)\right)}{t^{n-1}} .
\end{gathered}
$$

By the definition of $\widehat{\Omega}$ (see Theorem 5.1), from these inequalities we deduce that, if $\omega \in \widehat{\Omega}$, then $\tau_{z}(\omega) \in \widehat{\Omega}$ and

$$
\lim _{\substack{t \rightarrow+\infty \\ t \in \mathbb{Q}}} \frac{m_{G\left(\tau_{z}(\omega)\right)}^{\mathrm{pc}}\left(u_{0, \zeta, \nu}, Q_{t}^{v}(0)\right)}{t^{n-1}}=\lim _{\substack{t \rightarrow+\infty \\ t \in \mathbb{Q}}} \frac{m_{G(\omega)}^{\mathrm{pc}}\left(u_{0, \zeta, \nu}, Q_{t}^{\nu}(0)\right)}{t^{n-1}}
$$

This gives the desired inclusion $\tau_{z}(\widehat{\Omega}) \subset \widehat{\Omega}$.

The equality $P\left(\widetilde{\Omega} \cap \tau_{-z}(\widetilde{\Omega})\right)=1$ follows from the fact that $\tau_{-z}$ is measure perserving. If $\omega \in \widetilde{\Omega} \cap \tau_{-z}(\widetilde{\Omega})$, then $\omega, \tau_{z}(\omega) \in \widetilde{\Omega}$ and, by (5.1),

$$
\begin{aligned}
g_{\text {hom }}(\omega, \zeta, v) & =\lim _{t \rightarrow+\infty} \frac{m_{G(\omega)}^{\mathrm{pc}}\left(u_{0, \zeta, v}, Q_{t}^{v}(0)\right)}{t^{n-1}}, \\
g_{\text {hom }}\left(\tau_{z}(\omega), \zeta, v\right) & =\lim _{t \rightarrow+\infty} \frac{m_{G(\tau(\omega))}^{\mathrm{pc}}\left(u_{0, \zeta, v}, Q_{t}^{v}(0)\right)}{t^{n-1}} .
\end{aligned}
$$

By (6.6) this implies (6.3) for every $\omega \in \widetilde{\Omega} \cap \tau_{-z}(\widetilde{\Omega})$.

The next result shows that, in the ergodic case, the function $g_{\text {hom }}$ is independent of $\omega$.

Corollary 6.3. In addition to the assumptions of Theorem 6.2, suppose that the group $\left(\tau_{z}\right)_{z \in \mathbb{Z}^{n}}$ (resp. $\left.\left(\tau_{z}\right)_{z \in \mathbb{R}^{n}}\right)$ of P-preserving transformations on $(\Omega, \mathcal{T}, P)$ is ergodic. Then there exist a set $\widetilde{\Omega}_{0} \in \mathcal{T}$ with $\widetilde{\Omega}_{0} \subset \widetilde{\Omega}$ and $P\left(\widetilde{\Omega}_{0}\right)=1$, and a surface integrand $\tilde{g}_{\text {hom }} \in \mathcal{G}$, independent of $x$, such that $g_{\text {hom }}(\omega, \zeta, v)=\tilde{g}_{\text {hom }}(\zeta, v)$ for every $\omega \in \widetilde{\Omega}_{0}, \zeta \in \mathbb{R}_{0}^{m}$, and $\nu \in \mathbb{S}^{n-1}$. 
Proof. We start by showing that for every $\zeta \in \mathbb{Q}_{0}^{m}$ and $v \in \mathbb{Q}^{n} \cap \mathbb{S}^{n-1}$ there exist $\hat{g}_{\text {hom }}(\zeta, v) \in \mathbb{R}$ and a set $\widetilde{\Omega}^{\zeta, v} \in \mathcal{T}$, with $\widetilde{\Omega}^{\zeta, v} \subset \widetilde{\Omega}$ and $P\left(\widetilde{\Omega}^{\zeta, v}\right)=1$, such that

$$
g_{\text {hom }}(\omega, \zeta, v)=\hat{g}_{\text {hom }}(\zeta, v) \quad \text { for every } \omega \in \widetilde{\Omega}^{\zeta, v} \text {. }
$$

To this end we fix $\zeta \in \mathbb{Q}_{0}^{m}$ and $v \in \mathbb{Q}^{n} \cap \mathbb{S}^{n-1}$ and for every $c \in \mathbb{R}$ we define

$$
E_{c}^{\zeta, v}:=\left\{\omega \in \widetilde{\Omega}: g_{\text {hom }}(\omega, \zeta, v) \geq c\right\} .
$$

We now show that $P\left(\tau_{z}\left(E_{c}^{\zeta, v}\right) \triangle E_{c}^{\zeta, v}\right)=0$ for every $z \in \mathbb{Z}^{n}$ (resp. $z \in \mathbb{R}^{n}$ ). Note that the invariance follows by showing that $\omega \in \tau_{z}\left(E_{c}^{\zeta, v}\right)$ for $P$-almost everywhere $\omega \in E_{c}^{\zeta, v}$, the other inclusion being analogous. To see this, we first observe that for $P$-almost everywhere $\omega \in E_{c}^{\zeta, v}$ we have that $\omega \in E_{c}^{\zeta, v} \cap \tau_{z}(\widetilde{\Omega})$. Hence, by (6.3), we have that $\tau_{-z} \omega \in E_{c}^{\zeta, v}$, and equivalently that $\omega \in \tau_{z}\left(E_{c}^{\zeta, v}\right)$. Since $\left(\tau_{z}\right)_{z \in \mathbb{Z}^{n}}$ (resp. $\left.\left(\tau_{z}\right)_{z \in \mathbb{R}^{n}}\right)$ is ergodic, we can only have

$$
P\left(E_{c}^{\zeta, v}\right)=0 \text { or } P\left(E_{c}^{\zeta, v}\right)=1 .
$$

Since $E_{c_{1}}^{\zeta, \nu} \supset E_{c_{2}}^{\zeta, v}$ for $c_{1}<c_{2}$, by (6.7) there exists $c_{0}(\zeta, v) \in \mathbb{R}$ such that $P\left(E_{c}^{\zeta, v}\right)=0$ for $c>c_{0}(\zeta, v)$ and $P\left(E_{c}^{\zeta, v}\right)=1$ for $c<c_{0}(\zeta, v)$. It follows that there exists $\widetilde{\Omega}^{\zeta, v} \subset \widetilde{\Omega}$, with $P\left(\widetilde{\Omega}^{\zeta, v}\right)=1$, such that

$$
g_{\text {hom }}(\omega, \zeta, v)=c_{0}(\zeta, v) \quad \text { for every } \omega \in \widetilde{\Omega}^{\zeta, v}
$$

We define $\widetilde{\Omega}_{0}$ as the intersection of all sets $\widetilde{\Omega}^{\zeta, v}$ for $\zeta \in \mathbb{Q}_{0}^{m}$ and $v \in \mathbb{Q}^{n} \cap \mathbb{S}^{n-1}$. Then $\widetilde{\Omega}_{0} \subset \widetilde{\Omega}$ and $P\left(\widetilde{\Omega}_{0}\right)=1$. We now fix $\omega_{0} \in \widetilde{\Omega}_{0}$ and define $\tilde{g}_{\text {hom }}(\zeta, v):=$ $g_{\text {hom }}\left(\omega_{0}, \zeta, v\right)$ for every $\zeta \in \mathbb{R}_{0}^{m}$ and every $v \in \mathbb{Q}^{n} \cap \mathbb{S}^{n-1}$. By (6.8) we have

$$
g_{\text {hom }}(\omega, \zeta, v)=\tilde{g}_{\text {hom }}(\zeta, v) \text { for every } \omega \in \widetilde{\Omega}_{0}, \zeta \in \mathbb{Q}_{0}^{m}, v \in \mathbb{Q}^{n} \cap \mathbb{S}^{n-1} \text {. }
$$

The conclusion now follows from the continuity of $(\zeta, v) \mapsto g_{\text {hom }}(\omega, \zeta, v)$ on $\mathbb{R}_{0}^{m} \times \widehat{\mathbb{S}}_{ \pm}^{n-1}$ obtained in the proof of Theorem 5.1.

We now state some classical results from Probability Theory, which will be crucial for the proof of Theorem 6.1. For every $\psi \in L^{1}(\Omega, \mathcal{T}, P)$ and for every $\sigma$-algebra $\mathcal{T}^{\prime} \subset \mathcal{T}$, we will denote by $\mathbb{E}\left[\psi \mid \mathcal{T}^{\prime}\right]$ the conditional expectation of $\psi$ with respect to $\mathcal{T}^{\prime}$. This is the unique random variable in $L^{1}\left(\Omega, \mathcal{T}^{\prime}, P\right)$ with the property that

$$
\int_{E} \mathbb{E}\left[\psi \mid \mathcal{T}^{\prime}\right](\omega) d P(\omega)=\int_{E} \psi(\omega) \mathrm{d} P(\omega) \quad \text { for every } E \in \mathcal{T}^{\prime} .
$$

We start by stating Birkhoff's Ergodic Theorem (for a proof, see, for example, [29, Theorem 2.1.5]).

Theorem 6.4. [Birkhoff's Ergodic Theorem] Let $(\Omega, \mathcal{T}, P)$ be a probability space, let $T: \Omega \rightarrow \Omega$ be a $P$-preserving transformation, and let $\mathscr{I}_{P}(T)$ be the $\sigma$-algebra of $T$-invariants sets. Then for every $\psi \in L^{1}(\Omega, \mathcal{T}, P)$ we have

$$
\lim _{k \rightarrow+\infty} \frac{1}{k} \sum_{i=1}^{k} \psi\left(T^{i}(\omega)\right)=\mathbb{E}\left[\psi \mid \mathscr{I}_{P}(T)\right](\omega)
$$

for P-almost everywhere $\omega \in \Omega$. 
We also recall the Conditional Dominated Convergence Theorem, whose proof can be found in [12, Theorem 2.7].

Theorem 6.5. (Conditional Dominated Convergence). Let $\mathcal{T}^{\prime} \subset \mathcal{T}$ be a $\sigma$-algebra and let $\left(\varphi_{k}\right)$ be a sequence of random variables in $(\Omega, \mathcal{T}, P)$ converging pointwise $P$-almost everywhere in $\Omega$ to a random variable $\varphi$. Suppose that there exists $\psi \in$ $L^{1}(\Omega, \mathcal{T}, P)$ such that $\left|\varphi_{k}\right| \leq \psi P$-almost everywhere in $\Omega$ for every $k$. Then $\mathbb{E}\left[\varphi_{k} \mid \mathcal{T}^{\prime}\right](\omega) \rightarrow \mathbb{E}\left[\varphi \mid \mathcal{T}^{\prime}\right](\omega)$ for $P$-almost everywhere $\omega \in \Omega$.

We are now ready to prove the main result of this section.

Proof of Theorem 6.1. Let $g_{\text {hom }}$ and $\widetilde{\Omega}$ be as in Theorem 5.1. We will prove the existence of a set $\Omega^{\prime} \in \mathcal{T}$, with $\Omega^{\prime} \subset \widetilde{\Omega}$ and $P\left(\Omega^{\prime}\right)=1$, such that (6.1) holds for every $\omega \in \Omega^{\prime}$.

We only prove (6.1) in the case of a discrete group $\left(\tau_{z}\right)_{z \in \mathbb{Z}^{n}}$. If the set of indices is $\mathbb{R}^{n}$, the existence of $\Omega^{\prime}$ such that (6.1) holds in $\Omega^{\prime}$ can be proved by considering the restriction of the group to $\mathbb{Z}^{n}$.

In the following, for every $z \in \mathbb{Z}^{n}$ the sub- $\sigma$-algebra of invariant sets for the measure-preserving map $\tau_{z}$ is denoted by $\mathscr{I}_{z} \subset \mathcal{T}$; that is, $\mathscr{I}_{z}:=\{E \in \mathcal{T}$ : $\left.P\left(\tau_{z}(E) \triangle E\right)=0\right\}$. Also, for given $\zeta \in \mathbb{R}_{0}^{m}, v \in \mathbb{S}^{n-1}, \eta>0$, we define the sequence of events $\left(E_{j}^{\zeta, \nu, \eta}\right)_{j \in \mathbb{N}}$ as

$$
E_{j}^{\zeta, v, \eta}:=\left\{\omega \in \widetilde{\Omega}:\left|\frac{m_{G(\omega)}^{\mathrm{pc}}\left(u_{0, \zeta, v}, Q_{k}^{v}(0)\right)}{k^{n-1}}-g_{\mathrm{hom}}(\omega, \zeta, v)\right| \leq \eta \text { for every integer } k \geq j\right\} .
$$

We divide the proof into several steps. We use the notation for the integer part introduced in (q), Section 2.

Step 1. Let us fix $z \in \mathbb{Z}^{n}, \zeta \in \mathbb{R}_{0}^{m}, v \in \mathbb{S}^{n-1}$, and $\eta>0$. We prove that there exists a set $\widetilde{\Omega}_{z}^{\zeta, v, \eta} \in \mathcal{T}$, with $\widetilde{\Omega}_{z}^{\zeta, v, \eta} \subset \widetilde{\Omega}$ and $P\left(\widetilde{\Omega}_{z}^{\zeta, v, \eta}\right)=1$, satisfying the following property: for every $\delta>0$ and every $\omega \in \widetilde{\Omega}_{z}^{\zeta, \nu, \eta}$ there exists an integer $j_{0}=j_{0}(\zeta, v, \eta, z, \omega, \delta)$ such that

$$
\mathbb{E}\left[\chi_{E_{j_{0}}^{\zeta, v, \eta}} \mid \mathscr{I}_{z}\right](\omega)>1-\delta .
$$

To prove (6.9) we apply Theorem 5.1 and we obtain

$$
\lim _{j \rightarrow+\infty} \chi_{E_{j}^{\zeta, v, \eta}}(\omega)=1 \text { for every } \omega \in \widetilde{\Omega} .
$$

By the Conditional Dominated Convergence Theorem 6.5 there exists a set $\widetilde{\Omega}_{z}^{\zeta, \nu, \eta} \in$ $\mathcal{T}$, with $\widetilde{\Omega}_{z}^{\zeta, v, \eta} \subset \widetilde{\Omega}$ and $P\left(\widetilde{\Omega}_{z}^{\zeta, v, \eta}\right)=1$, such that

$$
\lim _{j \rightarrow+\infty} \mathbb{E}\left[\chi_{E_{j}^{\zeta, v, \eta}} \mid \mathscr{I}_{z}\right](\omega)=\mathbb{E}\left[1 \mid \mathscr{I}_{z}\right](\omega)=1 \text { for every } \omega \in \widetilde{\Omega}_{z}^{\zeta, v, \eta}
$$

Given $\omega \in \widetilde{\Omega}_{z}^{\zeta, \nu, \eta}$ and $\delta>0$, the existence of $j_{0}$ satisfying (6.9) follows from (6.10). 
Step 2. Let $z, \zeta, v$, and $\eta$ be as in Step 1 and let $0<\delta<\frac{1}{4}$. We prove that there exist a set $\Omega_{z}^{\zeta, v, \eta} \in \mathcal{T}$, with $\Omega_{z}^{\zeta, v, \eta} \subset \widetilde{\Omega}_{z}^{\zeta, v, \eta}$ and $P\left(\Omega_{z}^{\zeta, v, \eta}\right)=1$, and an integer $m_{0}=m_{0}(\zeta, v, \eta, z, \omega, \delta)>\frac{1}{\delta}$ satisfying the following property:

for every $\omega \in \Omega_{z}^{\zeta, v, \eta}$ and for every integer $m \geq m_{0}$ there exists $i=i(\zeta, v, \eta, z$, $\omega, \delta, m) \in\{m+1, \ldots, m+\ell\}$, with $\ell:=\lfloor 5 m \delta\rfloor$, such that

$$
\left|\frac{m_{G(\omega)}^{\mathrm{pc}}\left(u_{i z, \zeta, \nu}, Q_{k}^{v}(i z)\right)}{k^{n-1}}-g_{\text {hom }}(\omega, \zeta, v)\right| \leq \eta \quad \text { for every } k \geq j_{0},
$$

where $j_{0}=j_{0}(\zeta, v, \eta, z, \omega, \delta)$ is the integer introduced in Step 1.

To prove (6.11) we apply Birkhoff Ergodic Theorem 6.4 with $\psi:=\chi_{E_{j}^{\zeta, v, \eta}}$ and $T:=\tau_{z}$, and we obtain that there exists a set $\Omega_{z}^{\zeta, \nu, \eta} \in \mathcal{T}$, with $\Omega_{z}^{\zeta, \nu, \eta} \subset \widetilde{\Omega}_{z}^{\zeta, v, \eta}$ and $P\left(\Omega_{z}^{\zeta, v, \eta}\right)=1$, such that

$$
\lim _{m \rightarrow+\infty} \frac{1}{m} \sum_{i=1}^{m} \chi_{E_{j}^{\zeta, v, \eta}}\left(\tau_{i z}(\omega)\right)=\mathbb{E}\left[\chi_{E_{j}^{\zeta, v, \eta}} \mid \mathscr{I}_{z}\right](\omega)
$$

for every $j \in \mathbb{N}$ and every $\omega \in \Omega_{z}^{\zeta, \nu, \eta}$. In particular, for a given $\omega \in \Omega_{z}^{\zeta, \nu, \eta}$, equality (6.12) holds for the index $j_{0}=j_{0}(\zeta, v, \eta, z, \omega, \delta)$ introduced in Step 1. Therefore, there exists an integer $\hat{m}=\hat{m}(\zeta, v, \eta, z, \omega, \delta)$ such that

$$
\frac{1}{m} \sum_{i=1}^{m} \chi_{E_{j_{0}}^{\zeta, v, \eta}}\left(\tau_{i z}(\omega)\right)>\mathbb{E}\left[\chi_{E_{j_{0}}^{\zeta, v, \eta}} \mid \mathscr{I}_{z}\right](\omega)-\delta \quad \text { for every } m \geq \hat{m}
$$

Fix now an integer $m \geq m_{0}:=\max \left\{2 \hat{m}, 2 j_{0},\left\lfloor\frac{1}{\delta}\right\rfloor+1\right\}$ and set $\ell:=\lfloor 5 m \delta\rfloor$. We claim that

$$
\text { there exists } i=i(\zeta, v, \eta, z, \omega, \delta, m) \in\{m+1, \ldots, m+\ell\} \text { such that } \tau_{i z}(\omega) \in E_{j_{0}}^{\zeta, v, \eta} \text {. }
$$

Suppose, by contradiction, that (6.14) fails. Then, we have

$$
\begin{aligned}
\tilde{\ell} & :=\#\left\{i \in \mathbb{N}, 1 \leq i \leq m: \chi_{E_{j_{0}}^{\zeta, v, \eta}}\left(\tau_{i z}(\omega)\right)=1\right\} \\
& =\#\left\{i \in \mathbb{N}, 1 \leq i \leq m+\ell: \chi_{E_{j_{0}}^{\zeta, v, \eta}}\left(\tau_{i z}(\omega)\right)=1\right\} .
\end{aligned}
$$

So, (6.13) with $m$ replaced by $m+\ell$ gives

$$
\frac{\tilde{\ell}}{m+\ell}=\frac{1}{m+\ell} \sum_{i=1}^{m+\ell} \chi_{E_{j_{0}}^{\zeta, v, \eta}}\left(\tau_{i z}(\omega)\right)>\mathbb{E}\left[\chi_{E_{j_{0}}^{\zeta, v, \eta}} \mid \mathscr{I}_{z}\right](\omega)-\delta .
$$

Therefore, using (6.9) and (6.15) we obtain

$$
\begin{aligned}
\delta & >\mathbb{E}\left[\chi_{E_{j_{0}}^{\zeta, v, \eta}} \mid \mathscr{I}_{z}\right](\omega)-\frac{\tilde{\ell}}{m+\ell} \\
& =\mathbb{E}\left[\chi_{E_{j_{0}}^{\zeta, v, \eta}} \mid \mathscr{I}_{z}\right](\omega)-1+\frac{\ell+m-\tilde{\ell}}{m+\ell}>\frac{\ell+m-\tilde{\ell}}{m+\ell}-\delta .
\end{aligned}
$$


Since $m-\tilde{\ell} \geq 0$, from (6.16) we deduce that $\ell(1-2 \delta)<2 m \delta$. This, using the fact that $\delta<\overline{\frac{1}{4}}$, gives $\ell<4 m \delta$. On the other hand, by definition $\ell=\lfloor 5 m \delta\rfloor \geq$ $5 m \delta-1>4 m \delta$, since $m>\frac{1}{\delta}$. This contradicts the inequality $\ell<4 m \delta$ and proves (6.14). As a consequence, by the definition of $E_{j_{0}}^{\zeta, \nu, \eta}$,

$$
\left|\frac{m_{G\left(\tau_{i z}(\omega)\right)}^{\mathrm{pc}}\left(u_{0, \zeta, v}, Q_{k}^{v}(0)\right)}{k^{n-1}}-g_{\mathrm{hom}}\left(\tau_{i z}(\omega), \zeta, v\right)\right| \leq \eta
$$

for every integer $k \geq j_{0}$. Since $\omega \in \Omega_{z}^{\zeta, \nu, \eta} \subset \widetilde{\Omega}$, and $\tau_{i z}(\omega) \in E_{j_{0}}^{\zeta, v, \eta} \subset \widetilde{\Omega}$, thanks to (6.3) and (6.4) we get (6.11).

Step 3. We show that the result we want to prove is true along integers. More precisely, we prove that there exists $\Omega^{\prime} \in \mathcal{T}$, with $\Omega^{\prime} \subset \Omega$ and $P\left(\Omega^{\prime}\right)=1$, such that

$$
\lim _{\substack{k \rightarrow+\infty \\ k \in \mathbb{N}}} \frac{m_{G(\omega)}^{\mathrm{pc}}\left(u_{k z, \zeta, v}, Q_{m_{k}}^{v}(k z)\right)}{m_{k}^{n-1}}=g_{\text {hom }}(\omega, \zeta, v)
$$

for every $\omega \in \Omega^{\prime}, z \in \mathbb{Z}^{n}, \zeta \in \mathbb{Q}_{0}^{m}, v \in \mathbb{Q}^{n} \cap \mathbb{S}^{n-1}$, and for every sequence of integers $\left(m_{k}\right)$ such that $m_{k} \geq k$ for every $k$.

To prove this property, we define $\Omega^{\prime}$ as the intersection of the sets $\Omega_{z}^{\zeta, \nu, \eta}$ (introduced in Step 2) for $\zeta \in \mathbb{Q}_{0}^{m}, v \in \mathbb{Q}^{n} \cap \mathbb{S}^{n-1}, \eta \in \mathbb{Q}$, with $\eta>0$, and $z \in \mathbb{Z}^{n}$. It is clear that $\Omega^{\prime} \subset \widetilde{\Omega}$ and $P\left(\Omega^{\prime}\right)=1$. Let us fix $\omega, z, \zeta, v$ and $\left(m_{k}\right)$ as required. Moreover, let us fix $\delta>0$, with $20 \delta(|z|+1)<1$, and $\eta \in \mathbb{Q}$, with $\eta>0$. Let $m_{0}=m_{0}(\zeta, v, \eta, z, \omega, \delta)$ be as in Step 2 . For every $k \geq 2 m_{0}$ let $\underline{m}_{k}, \bar{m}_{k} \in \mathbb{Z}$ be defined as

$$
\underline{m}_{k}:=m_{k}-2\left(i_{k}-k\right)\lfloor|z|+1\rfloor \quad \text { and } \quad \bar{m}_{k}:=m_{k}+2\left(i_{k}-k\right)\lfloor|z|+1\rfloor,
$$

where $i_{k}=i(\zeta, v, \eta, z, \omega, \delta, k)$ is the index introduced in Step 2 corresponding to $m=k$. Clearly $\underline{m}_{k} \leq m_{k} \leq \bar{m}_{k}$. Moreover, since $|z|<\lfloor|z|+1\rfloor$, we have that

$$
Q_{\underline{m}_{k}}^{v}\left(i_{k} z\right) \subset \subset Q_{m_{k}}^{v}(k z) \subset \subset Q_{m_{k}}^{v}\left(i_{k} z\right)
$$

Let us now compare the minimisation problems for $G(\omega)$ relative to the cubes in (6.18). For every $k$ let $u_{k} \in S B V_{\mathrm{pc}}\left(Q_{m_{k}}^{v}(k z), \mathbb{R}^{m}\right)$ be such that with $u_{k}=u_{k z, \zeta, v}$ in a neighbourhood of $\partial Q_{m_{k}}^{v}(k z)$ and

$$
G(\omega)\left(u_{k}, Q_{m_{k}}^{v}(k z)\right) \leq m_{G(\omega)}^{\mathrm{pc}}\left(u_{k z, \zeta, v}, Q_{m_{k}}^{v}(k z)\right)+\eta
$$

thanks to (6.18) the extension of $u_{k}$ defined as

$$
v_{k}(y):= \begin{cases}u_{k}(y) & \text { if } y \in Q_{m_{k}}^{v}(k z), \\ u_{i_{k} z, \zeta, v}(y) & \text { if } y \in Q_{\bar{m}_{k}}^{v}\left(i_{k} z\right) \backslash Q_{m_{k}}^{v}(k z),\end{cases}
$$


belongs to $S B V_{\mathrm{pc}}\left(Q_{\bar{m}_{k}}^{v}\left(i_{k} z\right), \mathbb{R}^{m}\right)$ and satisfies $v_{k}=u_{i_{k} z, \zeta, v}$ in a neighbourhood of $\partial Q_{\bar{m}_{k}}^{v}\left(i_{k} z\right)$. By the definition of $v_{k}$ it follows that $S_{v_{k}} \subset S_{u_{k}} \cup \Sigma_{k}^{1} \cup \Sigma_{k}^{2}$, where

$$
\begin{aligned}
& \Sigma_{k}^{1}:=\left\{y \in \partial Q_{m_{k}}^{v}(k z):((y-k z) \cdot v)\left(\left(y-i_{k} z\right) \cdot v\right)<0\right\}, \\
& \Sigma_{k}^{2}:=\Pi_{-i_{k} z}^{v} \cap\left(Q_{\bar{m}_{k}}^{v}\left(i_{k} z\right) \backslash Q_{m_{k}}^{v}(k z)\right) .
\end{aligned}
$$

Moreover $\left|\left[v_{k}\right]\right|=|\zeta| \mathcal{H}^{n-1}$-almost everywhere on $\Sigma_{k}^{1} \cup \Sigma_{k}^{2}$. Since $20 \delta(|z|+1)<1$, $k \leq m_{k}$, and $i_{k}-k \leq 5 k \delta$ by (6.14), we obtain $\left|k z-i_{k} z\right| \leq\left(i_{k}-k\right)|z| \leq 5 k \delta|z| \leq$ $5 m_{k} \delta|z|<\frac{m_{k}}{2}$. Moreover, $\bar{m}_{k}-m_{k}=2\left(i_{k}-k\right)\lfloor|z|+1\rfloor \leq 10 k \delta\lfloor|z|+1\rfloor \leq$ $10 m_{k} \delta\lfloor|z|+1\rfloor<\frac{m_{k}}{2}$, hence $\bar{m}_{k}<2 m_{k}$. From the previous inequalities we obtain $\mathcal{H}^{n-1}\left(\Sigma_{k}^{1}\right) \leq 10(n-1) \delta|z| m_{k}^{n-1}$ and $\mathcal{H}^{n-1}\left(\Sigma_{k}^{2}\right)=\bar{m}_{k}^{n-1}-m_{k}^{n-1} \leq 5(n-$ 1) $2^{n-1} \delta\lfloor|z|+1\rfloor m_{k}^{n-1}$. Then by the growth condition $(g 6)$ we have

$$
G(\omega)\left(v_{k}, Q_{\bar{m}_{k}}^{v}\left(i_{k} z\right)\right) \leq G(\omega)\left(u_{k}, Q_{m_{k}}^{v}(k z)\right)+C_{\zeta, z} \delta m_{k}^{n-1},
$$

where $C_{\zeta, z}:=c_{5} 5(n-1)\left(2+2^{n-1}\right)\lfloor|z|+1\rfloor(1+|\zeta|)$. This inequality, combined with (6.19) and with the definition of $m_{G(\omega)}^{\mathrm{pc}}$, gives

$$
m_{G(\omega)}^{\mathrm{pc}}\left(u_{i_{k} z, \zeta, \nu}, Q_{\bar{m}_{k}}^{\nu}\left(i_{k} z\right)\right) \leq m_{G(\omega)}^{\mathrm{pc}}\left(u_{k z, \zeta, \nu}, Q_{m_{k}}^{\nu}(k z)\right)+\eta+C_{\zeta, z} \delta m_{k}^{n-1} .
$$

Thus, dividing all terms in (6.20) by $\bar{m}_{k}^{n-1}$ and recalling that $\bar{m}_{k} \geq m_{k}$, we get

$$
\frac{m_{G(\omega)}^{\mathrm{pc}}\left(u_{i_{k} z, \zeta, v}, Q_{\bar{m}_{k}}^{\nu}\left(i_{k} z\right)\right)}{\bar{m}_{k}^{n-1}} \leq \frac{m_{G(\omega)}^{\mathrm{pc}}\left(u_{k z, \zeta, v}, Q_{m_{k}}^{\nu}(k z)\right)}{m_{k}^{n-1}}+\frac{\eta}{m_{k}^{n-1}}+C_{\zeta, z} \delta .
$$

By the definition of $\underline{m}_{k}$ and since $m_{k} \geq \underline{m}_{k}$, a similar argument yields

$$
\frac{m_{G(\omega)}^{\mathrm{pc}}\left(u_{k z, \zeta, \nu}, Q_{m_{k}}^{\nu}(k z)\right)}{m_{k}^{n-1}} \leq \frac{m_{G(\omega)}^{\mathrm{pc}}\left(u_{i_{k} z, \zeta, v}, Q_{\underline{m}_{k}}^{\nu}\left(i_{k} z\right)\right)}{\underline{m}_{k}^{n-1}}+\frac{\eta}{m_{k}^{n-1}}+2 C_{\zeta, z} \delta .
$$

Since $\underline{m}_{k} \rightarrow+\infty$ as $k \rightarrow+\infty$ and $\bar{m}_{k} \geq \underline{m}_{k}$ for every $k$, we have $\bar{m}_{k} \geq \underline{m}_{k} \geq j_{0}$ for $k$ large enough, where $j_{0}=j_{0}(\zeta, v, \eta, z, \omega, \delta)$ is the integer introduced in Step 1. As $\omega \in \Omega_{z}^{\zeta, \nu, \eta}$, gathering (6.11), (6.21), and (6.22) gives

$$
\left|\frac{m_{G(\omega)}^{\mathrm{pc}}\left(u_{k z, \zeta, v}, Q_{m_{k}}^{v}(k z)\right)}{m_{k}^{n-1}}-g_{\text {hom }}(\omega, \zeta, v)\right| \leq \eta+\frac{\eta}{m_{k}^{n-1}}+2 C_{\zeta, z} \delta
$$

for $k$ large enough. We conclude that

$$
\limsup _{\substack{k \rightarrow+\infty \\ k \in \mathbb{N}}}\left|\frac{m_{G(\omega)}^{\mathrm{pc}}\left(u_{k z, \zeta, v}, Q_{m_{k}}^{v}(k z)\right)}{m_{k}^{n-1}}-g_{\mathrm{hom}}(\omega, \zeta, v)\right| \leq \eta+2 C_{\zeta, z} \delta .
$$


Since this inequality holds for every $\delta>0$, with $20 \delta(|z|+1)<1$, and every $\eta \in \mathbb{Q}$, with $\eta>0$, we obtain (6.17).

Step 4. We show that (6.1) holds when $\zeta$, and $v$ have rational coordinates. Namely, given $\omega \in \Omega^{\prime}$ (the set introduced in Step 3), $x \in \mathbb{R}^{n}, \zeta \in \mathbb{Q}_{0}^{m}, v \in \mathbb{Q}^{n} \cap \mathbb{S}^{n-1}$, and a function $r:(0,+\infty) \rightarrow(0,+\infty)$, with $r(t) \geq t$ for every $t>0$, we prove that (6.1) holds.

To this aim, we fix $\eta>0$. Then there exist $q \in \mathbb{Q}^{n}$ such that $|q-x|<\eta$ and $h \in \mathbb{N}$ such that $z:=h q \in \mathbb{Z}^{n}$.

Let $\left(t_{k}\right)$ be a sequence of real numbers with $t_{k} \rightarrow+\infty$ and let $s_{k}:=t_{k} / h$. By the definition of $m_{G(\omega)}^{\mathrm{pc}}$ for every $k$ there exists $\hat{u}_{k} \in S B V_{\mathrm{pc}}\left(Q_{r\left(t_{k}\right)}^{v}\left(t_{k} x\right), \mathbb{R}^{m}\right)$, with $\hat{u}_{k}=u_{t_{k} x, \zeta, v}$ in a neighbourhood of $\partial Q_{r\left(t_{k}\right)}^{v}\left(t_{k} x\right)$, such that

$$
G(\omega)\left(\hat{u}_{k}, Q_{r\left(t_{k}\right)}^{v}\left(t_{k} x\right)\right) \leq m_{G(\omega)}^{\mathrm{pc}}\left(u_{t_{k} x, \zeta, v}, Q_{r\left(t_{k}\right)}^{v}\left(t_{k} x\right)\right)+\eta
$$

We fix an integer $j>2|z|+1$ and define $r_{k}:=\left\lfloor r\left(t_{k}\right)+2 \eta t_{k}\right\rfloor+j$. It is easy to check that

$$
Q_{r\left(t_{k}\right)}^{v}\left(t_{k} x\right) \subset \subset Q_{r_{k}}^{v}\left(\left\lfloor s_{k}\right\rfloor z\right)
$$

As usual, we can extend $\hat{u}_{k}$ to $Q_{r_{k}}^{v}\left(\left\lfloor s_{k}\right\rfloor z\right)$ as

$$
\hat{v}_{k}(y):= \begin{cases}\hat{u}_{k}(y) & \text { if } y \in Q_{r\left(t_{k}\right)}^{v}\left(t_{k} x\right) \\ u_{\left\lfloor s_{k}\right\rfloor z, \zeta, v}(y) & \text { if } y \in Q_{r_{k}}^{v}\left(\left\lfloor s_{k}\right\rfloor z\right) \backslash Q_{r\left(t_{k}\right)}^{v}\left(t_{k} x\right) .\end{cases}
$$

Then $\hat{v}_{k} \in S B V_{\mathrm{pc}}\left(Q_{r_{k}}^{v}\left(\left\lfloor s_{k}\right\rfloor z\right), \mathbb{R}^{m}\right)$ and $\hat{v}_{k}=u_{\left\lfloor s_{k}\right\rfloor z, \zeta, v}$ in a neighbourhood of $\partial Q_{r_{k}}^{v}\left(\left\lfloor s_{k}\right\rfloor z\right)$. By the definition of $\hat{v}_{k}$ it follows that $S_{\hat{v}_{k}}=S_{\hat{u}_{k}} \cup \hat{\Sigma}_{k}^{1} \cup \hat{\Sigma}_{k}^{2}$, where

$$
\begin{aligned}
& \hat{\Sigma}_{k}^{1}:=\left\{y \in \partial Q_{r\left(t_{k}\right)}^{v}\left(t_{k} x\right):\left(\left(y-t_{k} x\right) \cdot v\right)\left(\left(y-\left\lfloor s_{k}\right\rfloor z\right) \cdot v\right)<0\right\}, \\
& \hat{\Sigma}_{k}^{2}:=\Pi_{\left\lfloor s_{k}\right\rfloor z}^{v} \cap\left(Q_{r_{k}}^{v}\left(\left\lfloor s_{k}\right\rfloor z\right) \backslash Q_{r\left(t_{k}\right)}^{v}\left(t_{k} x\right)\right) .
\end{aligned}
$$

Moreover $\left|\left[\hat{v}_{k}\right]\right|=|\zeta| \mathcal{H}^{n-1}$-almost everywhere on $\hat{\Sigma}_{k}^{1} \cup \hat{\Sigma}_{k}^{2}$. Since $\mid\left(t_{k} x-\right.$ $\left.\left\lfloor s_{k}\right\rfloor z\right) \cdot v|\leq| t_{k} x-t_{k} q|+| s_{k} z-\left\lfloor s_{k}\right\rfloor z\left|\leq t_{k} \eta+\right| z \mid$ we have $\mathcal{H}^{n-1}\left(\hat{\Sigma}_{k}^{1}\right) \leq$ $2(n-1) r\left(t_{k}\right)^{n-2}\left(t_{k} \eta+|z|\right)$ and $\mathcal{H}^{n-1}\left(\hat{\Sigma}_{k}^{2}\right)=r_{k}^{n-1}-r\left(t_{k}\right)^{n-1} \leq(n-1)\left(r\left(t_{k}\right)+\right.$ $\left.2 \eta t_{k}+j\right)^{n-2}\left(2 \eta t_{k}+j\right)$. Then by the growth conditions $(g 6)$ we have

$$
\begin{aligned}
G(\omega)\left(\hat{v}_{k}, Q_{r_{k}}^{v}\left(\left\lfloor s_{k}\right\rfloor z\right)\right) \leq & G(\omega)\left(\hat{u}_{k}, Q_{r\left(t_{k}\right)}^{v}\left(t_{k} x\right)\right) \\
& +C_{\zeta}\left(r\left(t_{k}\right)+2 \eta t_{k}+j\right)^{n-2}\left(2 \eta t_{k}+j\right),
\end{aligned}
$$

where $C_{\zeta}:=2(n-1) c_{5}(1+|\zeta|)$. This inequality, combined with (6.23) and with the definition of $m_{G(\omega)}^{\mathrm{pc}}$, gives

$$
\begin{aligned}
m_{G(\omega)}^{\mathrm{pc}}\left(u_{\left\lfloor s_{k}\right\rfloor z, \zeta, v}, Q_{r_{k}}^{v}\left(\left\lfloor s_{k}\right\rfloor z\right) \leq\right. & m_{G(\omega)}^{\mathrm{pc}}\left(u_{t_{k} x, \zeta, \nu}, Q_{r\left(t_{k}\right)}^{v}\left(t_{k} x\right)\right) \\
& +\eta+C_{\zeta}(1+3 \eta)^{n-2} 3 \eta r\left(t_{k}\right)^{n-1},
\end{aligned}
$$


for $k$ large enough so that $2 \eta t_{k}+j \leq 3 \eta r\left(t_{k}\right)$. Dividing all terms of the previous inequality by $r\left(t_{k}\right)^{n-1}$ and recalling that $r_{k} \geq r\left(t_{k}\right)$ we get

$$
\begin{aligned}
\frac{m_{G(\omega)}^{\mathrm{pc}}\left(u_{\left\lfloor s_{k}\right\rfloor z, \zeta, v}, Q_{r_{k}}^{v}\left(\left\lfloor s_{k}\right\rfloor z\right)\right.}{r_{k}^{n-1}} \leq & \frac{m_{G(\omega)}^{\mathrm{pc}}\left(u_{t_{k} x, \zeta, v}, Q_{r\left(t_{k}\right)}^{v}\left(t_{k} x\right)\right)}{r\left(t_{k}\right)^{n-1}} \\
& +\frac{\eta}{r\left(t_{k}\right)^{n-1}}+C_{\zeta}(1+3 \eta)^{n-2} 3 \eta .
\end{aligned}
$$

Finally, since $\omega \in \Omega^{\prime}, r_{k} \in \mathbb{N}, z \in \mathbb{Z}^{n}$, and $r_{k} \geq r\left(t_{k}\right) \geq t_{k} \geq s_{k} \geq\left\lfloor s_{k}\right\rfloor$, we can apply (6.17): By taking first the limit as $k \rightarrow+\infty$ and then as $\eta \rightarrow 0+$ we obtain

$$
g_{\text {hom }}(\omega, \zeta, v) \leq \liminf _{k \rightarrow+\infty} \frac{m_{G(\omega)}^{\mathrm{pc}}\left(u_{t_{k} x, \zeta, v}, Q_{r\left(t_{k}\right)}^{v}\left(t_{k} x\right)\right)}{r\left(t_{k}\right)^{n-1}} .
$$

A similar argument leads to

$$
\limsup _{k \rightarrow+\infty} \frac{m_{G(\omega)}^{\mathrm{pc}}\left(u_{t_{k} x, \zeta, v}, Q_{r\left(t_{k}\right)}^{v}\left(t_{k} x\right)\right)}{r\left(t_{k}\right)^{n-1}} \leq g_{\text {hom }}(\omega, \zeta, v),
$$

which, combined with (6.24), proves that (6.1) holds for every $\omega \in \Omega^{\prime}, x \in \mathbb{R}^{n}$, $\zeta \in \mathbb{Q}_{0}^{m}$, and $v \in \mathbb{Q}^{n} \cap \mathbb{S}^{n-1}$.

Step 5. We conclude the proof. We now extend this result to the general case $\zeta \in \mathbb{R}_{0}^{m}$ and $v \in \mathbb{S}^{n-1}$. To this end we fix $\omega \in \Omega^{\prime}$ and consider the functions $g(\omega, \cdot, \cdot, \cdot)$ and $\tilde{g}(\omega, \cdot, \cdot, \cdot)$ defined on $\mathbb{R}^{n} \times \mathbb{R}_{0}^{m} \times \mathbb{S}^{n-1}$ by $(5.10)$ and $(5.11)$, with $g(\cdot, \cdot, \cdot)$ replaced by $g(\omega, \cdot, \cdot, \cdot)$. In view of Step 4 we have

$$
\tilde{g}(\omega, x, \zeta, v)=\underset{g}{g}(\omega, x, \zeta, v)=g_{\text {hom }}(\omega, \zeta, v)
$$

for every $x \in \mathbb{R}^{n}, \zeta \in \mathbb{Q}_{0}^{m}$, and $v \in \mathbb{Q}^{n} \cap \mathbb{S}^{n-1}$. By Lemma 5.5 and arguing as in the last part of the proof of Theorem 5.1, we obtain that (6.25) holds for every $x \in \mathbb{R}^{n}, \zeta \in \mathbb{R}_{0}^{m}$, and $v \in \mathbb{S}^{n-1}$. This proves (6.1) for every $\omega \in \Omega^{\prime}, x \in \mathbb{R}^{n}$, $\zeta \in \mathbb{R}_{0}^{m}$, and $v \in \mathbb{S}^{n-1}$.

Moreover, if $\left(\tau_{z}\right)_{z \in \mathbb{Z}^{n}}$ (resp. $\left(\tau_{z}\right)_{z \in \mathbb{R}^{n}}$ ) is ergodic, then by Corollary 6.3 the function $g_{\text {hom }}$ does not depend on $\omega$ and (6.2) can be obtained by integrating (5.1) on $\Omega$, and using the Dominated Convergence Theorem thanks to (5.4).

Acknowledgements. F. Cagnetti wishes to thank Panagiotis E. Souganidis for suggesting to consider the stochastic counterpart of [15]. The authors are grateful to Marco Cicalese who drew their attention to the argument in [2, Proof of Theorem 5.5, Step 2] (see also the previous result by Braides and Piatnitski [14, Proposition 2.10]) which is crucial in the proof of Theorem 6.1. F. Cagnetti was supported by the EPSRC under the Grant EP/P007287/1 "Symmetry of Minimisers in Calculus of Variations". The research of G. Dal Maso was partially funded by the European Research Council under Grant No. 290888 "Quasistatic and Dynamic Evolution Problems in Plasticity and Fracture". G. Dal Maso is a member of the Gruppo Nazionale per l'Analisi Matematica, la Probabilità e le loro Applicazioni (GNAMPA) of the Istituto Nazionale di Alta Matematica (INdAM). L. Scardia acknowledges support by the EPSRC under the Grant EP/N035631/1 "Dislocation patterns beyond optimality". 
Open Access This article is distributed under the terms of the Creative Commons Attribution 4.0 International License (http://creativecommons.org/licenses/by/4.0/), which permits unrestricted use, distribution, and reproduction in any medium, provided you give appropriate credit to the original author(s) and the source, provide a link to the Creative Commons license, and indicate if changes were made.

Publisher's Note Springer Nature remains neutral with regard to jurisdictional claims in published maps and institutional affiliations.

\section{Compliance with Ethical Standards}

Conflict of interest: The authors declare to have no conflict of interest.

\section{Appendix. Measurability Issues}

The main result of this section is the following proposition, which gives the measurability of the function $\omega \mapsto m_{G(\omega)}^{\mathrm{pc}}(w, A)$. This property was crucial in the proof of Proposition 5.3.

Proposition A.1. Let $(\Omega, \widehat{\mathcal{T}}, \widehat{P})$ be the completion of the probability space $(\Omega, \mathcal{T}$, $P)$, let $g$ be a random surface integrand, and let $A \in \mathscr{A}$. Let $G(\omega)$ be as in (3.2), with $g(\cdot, \cdot)$ replaced by $g(\omega, \cdot, \cdot, \cdot)$. Let $w \in L^{0}\left(\mathbb{R}^{n}, \mathbb{R}^{m}\right)$ be such that $\left.w\right|_{A} \in$ $S B V_{\mathrm{pc}}\left(A, \mathbb{R}^{m}\right) \cap L^{\infty}\left(A, \mathbb{R}^{m}\right)$, and for every $\omega \in \Omega$ let $m_{G(\omega)}^{\mathrm{pc}}(w, A)$ be as in (3.4), with $G$ replaced by $G(\omega)$. Then the function $\omega \mapsto m_{G(\omega)}^{\mathrm{pc}}(w, A)$ is $\widehat{\mathcal{T}}$-measurable.

The main difficulty in the proof of Proposition A.1 is that, although $\omega \mapsto$ $G(\omega)(u, A)$ is clearly $\mathcal{T}$-measurable, $m_{G(\omega)}^{\mathrm{pc}}(w, A)$ is defined as an infimum on an uncountable set. This difficulty is usually solved by means of the Projection Theorem, which requires the completeness of the probability space. It also requires joint measurability in $(\omega, u)$ and some topological properties of the space on which the infimum is taken, like separability and metrisability. In our case (see (3.4)) the infimum is taken on the space of all functions $u \in L^{0}\left(\mathbb{R}^{n}, \mathbb{R}^{m}\right)$ such that $\left.u\right|_{A} \in S B V_{\mathrm{pc}}\left(A, \mathbb{R}^{m}\right)$ and $u=w$ near $\partial A$, and it is not easy to find a topology on this space with the above mentioned properties and such that $(\omega, u) \mapsto G(\omega)(u, A)$ is jointly measurable. Therefore we have to attack the measurability problem in an indirect way, extending (an approximation of) $G(\omega)(u, A)$ to a suitable subset of the space of bounded Radon measures, which turns out to be compact and metrisable in the weak* topology.

We start by introducing some notation that will be used later. For every every $A \in \mathscr{A}$ we denote by $\mathcal{M}_{b}\left(A, \mathbb{R}^{m \times n}\right)$ the Banach space of all $\mathbb{R}^{m \times n}$-valued Radon measures on $A$. This space is identified with the dual of the space $C_{0}\left(A, \mathbb{R}^{m \times n}\right)$ of all $\mathbb{R}^{m \times n}$-valued continuous functions on $\bar{A}$ vanishing on $\partial A$. For every $R>0$ we set

$$
\mathcal{M}_{A}^{R}:=\left\{\mu \in \mathcal{M}_{b}\left(A, \mathbb{R}^{m \times n}\right):|\mu|(A) \leq R\right\},
$$

where $|\mu|$ denotes the variation of $\mu$ with respect to the Euclidean norm on $\mathbb{R}^{m \times n}$. On $\mathcal{M}_{A}^{R}$ we consider the topology induced by the weak topology of $\mathcal{M}_{b}\left(A, \mathbb{R}^{m \times n}\right)$. Before starting the proof of Proposition A.1, we need two preliminary results. 
Lemma A.2. Let $(\Lambda, \mathcal{S})$ be a measurable space, let $A \in \mathscr{A}$, let $R>0$, and let $h: \Lambda \times A \rightarrow \mathbb{R}$ be a bounded and $\mathcal{S} \otimes \mathscr{B}(A)$-measurable function. Let $H: \Lambda \times$ $\mathcal{M}_{A}^{R} \rightarrow \mathbb{R}$ be defined by

$$
H(\lambda, \mu):=\int_{A} h(\lambda, x) \mathrm{d}|\mu|(x) .
$$

Then $H$ is $\mathcal{S} \otimes \mathscr{B}\left(\mathcal{M}_{A}^{R}\right)$-measurable.

Proof. Let $\mathcal{H}$ be the set of all bounded, $\mathcal{S} \otimes \mathscr{B}(A)$-measurable functions $h$ such that the function $H$ defined by (A.26) is $\mathcal{S} \otimes \mathscr{B}\left(\mathcal{M}_{A}^{R}\right)$-measurable. Clearly $\mathcal{H}$ is a monotone class (see, for example, [3, Definition 4.12]) which contains all the functions of the form $h(\lambda, x)=\varphi(\lambda) \psi(x)$ with $\varphi$ bounded and $\mathcal{S}$-measurable and $\psi \in C_{c}^{0}(A)$. Then the functional form of the Monotone Class Theorem (see, for example, [23, Chapter I, Theorem 21]) implies that $\mathcal{H}$ coincides with the class of all bounded and $\mathcal{S} \otimes \mathscr{B}(A)$-measurable functions and this concludes the proof.

Corollary A.3. Let $A \in \mathscr{A}$, let $R>0$, and let $h: \Omega \times A \times \mathcal{M}_{A}^{R} \rightarrow \mathbb{R}$ be a bounded and $\mathcal{T} \otimes \mathscr{B}(A) \otimes \mathscr{B}\left(\mathcal{M}_{A}^{R}\right)$-measurable function. Let $H: \Omega \times \mathcal{M}_{A}^{R} \rightarrow \mathbb{R}$ be defined by

$$
H(\omega, \mu):=\int_{A} h(\omega, x, \mu) \mathrm{d}|\mu|(x) .
$$

Then $H$ is $\mathcal{T} \otimes \mathscr{B}\left(\mathcal{M}_{A}^{R}\right)$-measurable.

Proof. As a preliminary step, we consider the augmented functional $\tilde{H}: \Omega \times M_{A}^{R} \times$ $\mathcal{M}_{A}^{R} \rightarrow \mathbb{R}$ defined by

$$
\tilde{H}(\omega, v, \mu):=\int_{A} h(\omega, x, v) \mathrm{d}|\mu|(x) .
$$

By applying Lemma A.2 to $\tilde{H}$, with $\Lambda=\Omega \times \mathcal{M}_{A}^{R}, \lambda=(\omega, v)$, and $\mathcal{S}=\mathcal{T} \otimes$ $\mathscr{B}\left(\mathcal{M}_{A}^{R}\right)$, we deduce that $\tilde{H}$ is $\mathcal{T} \otimes \mathscr{B}\left(\mathcal{M}_{A}^{R}\right) \otimes \mathscr{B}\left(\mathcal{M}_{A}^{R}\right)$-measurable.

The claim then follows by noting that $H(\omega, \mu)=\tilde{H}(\omega, \mu, \mu)$ and by observing that $(\omega, \mu) \mapsto(\omega, \mu, \mu)$ is measurable for the $\sigma$-algebras $\mathcal{T} \otimes \mathscr{B}\left(\mathcal{M}_{A}^{R}\right)$ and $\mathcal{T} \otimes$ $\mathscr{B}\left(\mathcal{M}_{A}^{R}\right) \otimes \mathscr{B}\left(\mathcal{M}_{A}^{R}\right)$.

We are now ready to give the proof of Proposition A.1.

Proof of Proposition A.1. For every $k \in \mathbb{N}$ let $m_{G(\omega)}^{k}(w, A)$ be as in (3.6), with $G$ replaced by $G(\omega)$. In view of (3.5), the function $\omega \mapsto m_{G(\omega)}(w, A)$ is $\widehat{\mathcal{T}}$ measurable if

$$
\omega \mapsto m_{G(\omega)}^{k}(w, A) \text { is } \widehat{\mathcal{T}} \text {-measurable }
$$

for $k$ sufficiently large. To prove this property we fix $k>\|w\|_{L^{\infty}\left(A, \mathbb{R}^{m}\right)}$ and observe that there is a one-to-one correspondence between the space of rank one $m \times n$ matrices and the quotient of $\mathbb{R}_{0}^{m} \times \mathbb{S}^{n-1}$ with respect to the equivalence relation 
$(\zeta, v) \sim(-\zeta,-v)$. Therefore, thanks to $(g 6)$ and $(g 7)$, for every $k \in \mathbb{N}$ we can define a bounded $\mathcal{T} \otimes \mathscr{B}(A) \otimes \mathscr{B}^{m \times n}$-measurable function $\tilde{g}_{k}: \Omega \times A \times \mathbb{R}^{m \times n} \rightarrow \mathbb{R}$ such that

$$
\tilde{g}_{k}(\omega, x, \zeta \otimes \nu)=g(\omega, x, \zeta, v)
$$

for every $\omega \in \Omega, x \in A, \zeta \in \mathbb{R}_{0}^{m}$ with $|\zeta| \leq 2 k, v \in \mathbb{S}^{n-1}$. This implies that

$$
\begin{aligned}
G(\omega)(u, A) & =\int_{S_{u} \cap A} g\left(\omega, x,[u], v_{u}\right) \mathrm{d} \mathcal{H}^{n-1} \\
& =\int_{S_{u} \cap A} \tilde{g}_{k}\left(\omega, x,[u] \otimes v_{u}\right) \mathrm{d} \mathcal{H}^{n-1}
\end{aligned}
$$

for every $u \in S B V\left(A, \mathbb{R}^{m}\right) \cap L^{\infty}\left(A, \mathbb{R}^{m}\right)$ with $\|u\|_{L^{\infty}\left(A, \mathbb{R}^{m}\right)} \leq k$.

Let $\alpha:=c_{5} / c_{4}\left(1+2\|w\|_{L^{\infty}\left(A, \mathbb{R}^{m}\right)}\right) \mathcal{H}^{n-1}\left(S_{w} \cap A\right)$ as in Remark 3.4. Given an increasing sequence $\left(A_{j}\right)$ of open sets, with $A_{j} \subset \subset A$ and $A_{j} \nearrow A$, we define $\mathcal{X}_{j}^{k}:=\left\{u \in L^{0}\left(\mathbb{R}^{n}, \mathbb{R}^{m}\right):\left.u\right|_{A} \in S B V_{\mathrm{pc}}\left(A, \mathbb{R}^{m}\right) \cap L^{\infty}\left(A, \mathbb{R}^{m}\right)\right.$,

$$
\left.\|u\|_{L^{\infty}\left(A, \mathbb{R}^{m}\right)} \leq k, \mathcal{H}^{n-1}\left(S_{u} \cap A\right) \leq \alpha, u=w \text { in } A \backslash A_{j}\right\} .
$$

By (3.6) we have

$$
\lim _{j \rightarrow+\infty} \inf _{u \in \mathcal{X}_{j}^{k}} G(\omega)(u, A)=m_{G(\omega)}^{k}(w, A) .
$$

Therefore, to prove (A.27), and hence the $\widehat{\mathcal{T}}$-measurability of $\omega \mapsto m_{G(\omega)}^{\mathrm{pc}}(w, A)$ it is enough to show that

$$
\omega \mapsto \inf _{u \in \mathcal{X}_{j}^{k}} G(\omega)(u, A) \text { is } \widehat{\mathcal{T}} \text {-measurable. }
$$

This will be obtained by using the Projection Theorem. To this end we consider $\mathcal{X}_{j}^{k}$ as a topological space, with the topology induced by the weak ${ }^{*}$-topology of $B V\left(A, \mathbb{R}^{m}\right)$, which is metrisable on $\mathcal{X}_{j}^{k}$. Indeed $B V\left(A, \mathbb{R}^{m}\right)$ is the dual of a separable space (see [4, Remark 3.12]), and $\mathcal{X}_{j}^{k}$ is bounded with respect to the $B V\left(A, \mathbb{R}^{m}\right)$ norm, since every $u \in \mathcal{X}_{j}^{k}$ satisfies

$$
\|u\|_{B V\left(A, \mathbb{R}^{m}\right)}=\|u\|_{L^{1}\left(A, \mathbb{R}^{m}\right)}+|D u|(A) \leq k \mathcal{L}^{n}(A)+2 k \alpha .
$$

Further, by virtue of Ambrosio's Compactness Theorem for $S B V\left(A, \mathbb{R}^{m}\right)$ (see [4, Theorem 4.8]), the topological space $\mathcal{X}_{j}^{k}$ is compact.

Let $\pi_{\Omega}: \Omega \times \mathcal{X}_{j}^{k} \rightarrow \Omega$ be the canonical projection of $\Omega \times \mathcal{X}_{j}^{k}$ onto $\Omega$. For every $t \in \mathbb{R}$ we have

$\left\{\omega \in \Omega: \inf _{u \in \mathcal{X}_{j}^{k}} G(\omega)(u, A)<t\right\}=\pi_{\Omega}\left(\left\{(\omega, u) \in \Omega \times \mathcal{X}_{j}^{k}: G(\omega)(u, A)<t\right\}\right)$. 
By the Projection Theorem (see, for example, [23, Theorem III.13 and 33(a)]), (A.29) follows if we show that

$$
(\omega, u) \mapsto G(\omega)(u, A) \text { is } \mathcal{T} \otimes \mathscr{B}\left(\mathcal{X}_{j}^{k}\right) \text {-measurable, }
$$

hence $\widehat{\mathcal{T}} \otimes \mathscr{B}\left(\mathcal{X}_{j}^{k}\right)$-measurable.

To prove this property we shall use (A.28). By a Monotone Class argument (see the proof of Lemma A.2) we can assume, without loss of generality, that for every $\omega \in \Omega$ and every $x \in \mathbb{R}^{n}$ the function $\xi \mapsto \tilde{g}_{k}(\omega, x, \xi)$ is continuous.

In (A.28) it is convenient to express $[u] \otimes v_{u}$ and the restriction of $\mathcal{H}^{n-1}$ to $S_{u}$ by means of the measure $\mu:=D u$. By [4, Theorems 3.77 and 3.78] for every $B \in \mathscr{B}(A)$ we have

$$
\mu(B)=\int_{S_{u} \cap B}[u] \otimes v_{u} \mathrm{~d} \mathcal{H}^{n-1} \text { and }|\mu|(B)=\int_{S_{u} \cap B}|[u]| \mathrm{d} \mathcal{H}^{n-1},
$$

hence

$$
\mathcal{H}^{n-1}(B)=\int_{S_{u} \cap B} \frac{1}{|[u]|} \mathrm{d}|\mu| .
$$

To write (A.28) as a limit of measurable functions, for every $\mu \in \mathcal{M}_{b}\left(A, \mathbb{R}^{m \times n}\right)$ and $\rho>0$ we consider the measure $\mu^{\rho} \in \mathcal{M}_{b}\left(A, \mathbb{R}^{m \times n}\right)$ defined by

$$
\mu^{\rho}(B):=\frac{\mu(B)}{\omega_{n-1} \rho^{n-1}} \text { for every } B \in \mathscr{B}(A),
$$

where $\omega_{n-1}$ is the measure of the unit ball in $\mathbb{R}^{n-1}$. If $u \in S B V_{\mathrm{pc}}\left(A, \mathbb{R}^{m}\right)$ and $\mu=D u$, by the Besicovich Derivation Theorem and by the rectifiability of $S_{u}$ (see [4, Theorems 2.22, 2.83, and 3.78]) we deduce from (A.31) that, when $\rho \rightarrow 0+$,

$$
\mu^{\rho}\left(B_{\rho}(x) \cap A\right) \rightarrow\left([u] \otimes v_{u}\right)(x) \text { for } \mathcal{H}^{n-1} \text {-almost everywhere } x \in S_{u} \cap A,
$$

$$
\left|\mu^{\rho}\right|\left(B_{\rho}(x) \cap A\right) \rightarrow|[u](x)| \text { for } \mathcal{H}^{n-1} \text {-almost everywhere } x \in S_{u} \cap A .
$$

Since $\xi \mapsto \tilde{g}_{k}(\omega, x, \xi)$ is continuous and bounded uniformly with respect to $x$, by the Dominated Convergence Theorem it follows from (A.32), (A.33), and (A.34) that for every $u \in \mathcal{X}_{j}^{k}$ we have

$$
G(\omega)(u, A)=\lim _{\eta \rightarrow 0+\rho \rightarrow 0+} \lim _{A} \frac{\tilde{g}_{k}\left(\omega, x, \mu^{\rho}\left(A \cap B_{\rho}(x)\right)\right)}{\max \left\{\left|\mu^{\rho}\right|\left(A \cap B_{\rho}(x)\right), \eta\right\}} \mathrm{d}|\mu|(x),
$$

with $\mu:=D u$. Let $R:=2 k \alpha$. Since the map $u \mapsto D u$ from $B V\left(A, \mathbb{R}^{m}\right)$ into $\mathcal{M}_{b}\left(A, \mathbb{R}^{m \times n}\right)$ is continuous for the weak* topologies and the image of $\mathcal{X}_{j}^{k}$ under this map is contained in $\mathcal{M}_{A}^{R}$, the claim in (A.30) is an obvious consequence of (A.35) and of the following property: for every $\eta>0$ and $\rho>0$ the function $(\omega, \mu) \mapsto \int_{A} \frac{\tilde{g}_{k}\left(\omega, x, \mu^{\rho}\left(A \cap B_{\rho}(x)\right)\right)}{\max \left\{\left|\mu^{\rho}\right|\left(A \cap B_{\rho}(x)\right), \eta\right\}} \mathrm{d}|\mu|(x)$ is $\mathcal{T} \otimes \mathscr{B}\left(\mathcal{M}_{A}^{R}\right)$-measurable. 
To prove this property we observe that

$$
(x, \mu) \mapsto\left|\mu^{\rho}\right|\left(A \cap B_{\rho}(x)\right) \text { is (jointly) lower semicontinuous on } A \times \mathcal{M}_{A}^{R} \text {. }
$$

This is a consequence of the equality

$$
|\mu|\left(B_{\rho}(x) \cap A\right)=\sup \left\{\int_{A} \varphi(y-x) \mathrm{d} \mu(y): \varphi \in C_{c}^{1}\left(B_{\rho}(0), \mathbb{R}^{m \times n}\right),|\varphi| \leq 1\right\}
$$

and of the (joint) continuity of $(x, \mu) \mapsto \int_{A} \varphi(y-x) \mathrm{d} \mu(y)$ on $A \times \mathcal{M}_{A}^{R}$.

We also observe that the $\mathbb{R}^{m \times n}$-valued function

$$
(x, \mu) \mapsto \mu^{\rho}\left(A \cap B_{\rho}(x)\right) \text { is } \mathscr{B}(A) \otimes \mathscr{B}\left(\mathcal{M}_{A}^{R}\right) \text {-measurable. }
$$

Indeed, given a nondecreasing sequence $\left(\varphi_{j}\right)$ of nonnegative functions in $C_{c}^{1}\left(B_{\rho}(0)\right)$ converging to 1 , we have

$$
\mu^{\rho}\left(A \cap B_{\rho}(x)\right)=\frac{1}{\omega_{n-1} \rho^{n-1}} \lim _{j \rightarrow+\infty} \int_{A} \varphi_{j}(y-x) \mathrm{d} \mu(y),
$$

and each function $(x, \mu) \mapsto \int_{A} \varphi_{j}(y-x) \mathrm{d} \mu(y)$ is (jointly) continuous on $A \times \mathcal{M}_{A}^{R}$. Since $\tilde{g}_{k}$ is $\mathcal{T} \otimes \mathscr{B}(A) \otimes \mathscr{B}^{m \times n}$-measurable, from (A.37) and (A.38) we obtain that $(\omega, x, \mu) \mapsto \frac{\tilde{g}_{k}\left(\omega, x, \mu^{\rho}\left(A \cap B_{\rho}(x)\right)\right)}{\max \left\{\left|\mu^{\rho}\right|\left(A \cap B_{\rho}(x)\right), \eta\right\}}$ is $\mathcal{T} \otimes \mathscr{B}(A) \otimes \mathscr{B}\left(\mathcal{M}_{A}^{R}\right)$-measurable,

and (A.36) follows from Corollary A.3. This concludes the proof.

\section{References}

1. Akcoglu, M.A., Krengel, U.: Ergodic theorems for superadditive processes. J. Reine Angew. Math. 323, 53-67, 1981

2. Alicandro, R., Cicalese, M., Ruf, M.: Domain formation in magnetic polymer composites: an approach via stochastic homogenization. Arch. Rat. Mech. Anal. 218, 945984, 2015

3. Aliprantis, C.D., Border, K.C.: Infinite Dimensional Analysis. Springer, Berlin, 2006

4. Ambrosio, L., Fusco, N., Pallara, D.: Functions of Bounded Variations and Free Discontinuity Problems. Clarendon Press, Oxford, 2000

5. Armstrong, S.N., Kuusi, T., Mourrat, J.-C.: Mesoscopic higher regularity and subadditivity in elliptic homogenization. Commun. Math. Phys. 347(2), 315-361, 2016

6. Armstrong, S.N., Kuusi, T., Mourrat, J.-C.: The additive structure of elliptic homogenization. Invent. Math. 208(3), 999-1054, 2017

7. Armstrong, S.N., Mourrat, J.-C.: Lipschitz regularity for elliptic equations with random coefficients. Arch. Ration. Mech. Anal. 219(1), 255-348, 2016

8. Armstrong, S.N., Smart, C.K.: Quantitative stochastic homogenization of convex integral functionals. Ann. Sci. Éc. Norm. Supér. (4) 49(2), 423-481, 2016

9. Barchiesi, M., Dal Maso, G.: Homogenization of fiber reinforced brittle materials: the extremal cases. SIAM J. Math. Anal. 41(5), 1874-1889, 2009

10. Barchiesi, M., Focardi, M.: Homogenization of the Neumann problem in perforated domains: an alternative approach. Calc. Var. Partial Differ. Equ. 42, 257-288, 2011 
11. Barchiesi, M., LazZaroni, G., ZePPIERI, C.I.: A bridging mechanism in the homogenisation of brittle composites with soft inclusions. SIAM J. Math. Anal. 48(2), 1178-1209, 2016

12. Bhattacharya, R., Waymire, E.C.: A Basic Course in Probability Theory. Springer, Berlin, 2007

13. Braides, A., Defranceschi, A., Vitali, E.: Homogenization of free discontinuity problems. Arch. Ration. Mech. Anal. 135, 297-356, 1996

14. Braides, A., Piatnitski, A.: Homogenization of surface and length energies for spin systems. J. Funct. Anal. 264, 1296-1328, 2013

15. Cagnetti, F., Scardia, L.: An extension theorem in $S B V$ and an application to the homogenization of the Mumford-Shah functional in perforated domains. J. Math. Pures Appl. 95, 349-381, 2011

16. Cagnetti, F., Dal Maso, G., Scardia L., Zeppieri, C. I.: $\Gamma$-Convergence of freediscontinuity problems. Ann. Inst. H. Poincaré Anal. Non Linéaire. Published online at https://doi.org/10.1016/j.anihpc.2018.11.003

17. Dal Maso, G., Modica, L.: Nonlinear stochastic homogenization. Ann. Mat. Pura Appl. (4) 144, 347-389, 1986

18. Dal Maso, G., Modica, L.: Nonlinear stochastic homogenization and ergodic theory. J. Reine Angew. Math. 368, 28-42, 1986

19. Dal Maso, G., Francfort, G.A., Toader, R.: Quasistatic crack growth in nonlinear elasticity. Arch. Ration. Mech. Anal. 176, 165-225, 2005

20. Dal Maso, G., Zeppieri, C.I.: Homogenization of fiber reinforced brittle materials: the intermediate case. Adv. Calc. Var. 3(4), 345-370, 2010

21. De Giongi, E.: Free Discontinuity problems in calculus of variations. Frontiers in pure and applied Mathematics, a collection of papers dedicated to J.L. Lions on the occasion of his 60th birthday, R. Dautray ed., North Holland, 1991

22. De Giorgi, E., Ambrosio, L.: New functionals in the calculus of variations. Atti Accad. Naz. Lincei Rend. Cl. Sci. Fis. Mat. Natur. (8) 82(2), (1988) 199-210, 1989 (Italian)

23. Dellacherie, C., Meyer, P.-A.: Probabilities and Potential. North-Holland Publishing Company, Amsterdam, 1978

24. Doob, J.L.: Stochastic Processes. John Wiley \& Sons, Wiley Classics Library Edition, Hoboken, 1990

25. Focardi, M., Gelli, M.S., Ponsiglione, M.: Fracture mechanics in perforated domains: a variational model for brittle porous media. Math. Models Methods Appl. Sci. 19, 2065-2100, 2009

26. Gloria, A., Отto, F.: An optimal variance estimate in stochastic homogenization of discrete elliptic equations. Ann. Probab. 39(3), 779-856, 2011

27. Gloria, A., Otto, F.: An optimal error estimate in stochastic homogenization of discrete elliptic equations. Ann. Appl. Probab. 22(1), 1-28, 2012

28. Kozlov, S. M.: The averaging of random operators. Mat. Sb. 109, 188-2012, 1979

29. Krengel, U.: Ergodic theorems. De Gruyter Studies in Mathematics, Vol. 6. Walter de Gruyter \& Co., Berlin, 1985

30. Messaoudi, K., Michaille, G.: Stochastic homogenization of nonconvex integral functionals. RAIRO Modél. Math. Anal. Numér. (3) 28, 329-356, 1991

31. Papanicolaou, G.C., Varadhan, S.R.S.: Boundary value problems with rapidly oscillating random coefficients. Random Fields, Vol. I, II (Esztergom, 1979). Colloquia Mathematica Societatis János Bolyai, 27. North-Holland, Amsterdam, 1981

32. Papanicolaou, G.C., Varadhan, S.R.S.: Diffusions with random coefficients. Statistics and Probability: Essays in Honor of C. R. Rao. North-Holland, Amsterdam, 1982

33. Pellet, X., Scardia, L., Zeppieri, C. I.: Homogenization of high-contrast MumfordShah energies, submitted 2018. Preprint version: arXiv:1807.08705

34. Scardia, L.: Damage as $\Gamma$-limit of microfractures in anti-plane linearized elasticity. Math. Models Methods Appl. Sci. 18, 1703-1740, 2008

35. Scardia, L.: Damage as the $\Gamma$-limit of microfractures in linearized elasticity under the non-interpenetration constraint. Adv. Calc. Var. 3, 423-458, 2010 
Filippo Cagnetti

Department of Mathematics,

University of Sussex,

Brighton,

UK.

e-mail: F.Cagnetti@sussex.ac.uk

and

\author{
Gianni Dal Maso \\ SISSA, \\ Trieste, \\ Italy. \\ e-mail: dalmaso@sissa.it \\ and \\ Lucia SCARDia \\ Department of Mathematics, \\ Heriot-Watt University, \\ Edinburgh, \\ UK. \\ e-mail: L.Scardia@hw.ac.uk \\ and \\ CATERINa Ida ZePPIERI \\ WWU Münster, \\ Münster, \\ Germany. \\ e-mail: caterina.zeppieri@uni-muenster.de
}

Institut für numerische und angewandte Mathematik,

(Received February 2, 2018 / Accepted March 13, 2019)

Published online March 28, 2019

(C) The Author(s) (2019) 